\title{
Analytic structure of all loop banana integrals
}

\author{
Kilian Bönisch, ${ }^{a}$ Fabian Fischbach, ${ }^{a}$ Albrecht Klemm, ${ }^{a, b}$ Christoph Nega ${ }^{a, 1}$ \\ and Reza Safari ${ }^{a}$ \\ ${ }^{a}$ Bethe Center for Theoretical Physics, \\ Universität Bonn, D-53115, Germany \\ ${ }^{b}$ Hausdorff Center for Mathematics, \\ Universität Bonn, D-53115, Germany \\ E-mail: boenisch@th.physik.uni-bonn.de, fischbach@physik.uni-bonn.de, \\ aklemm@th.physik.uni-bonn.de, cnega@th.physik.uni-bonn.de, \\ rsafari@th.physik. uni-bonn.de
}

ABstract: Using the Gelfand-Kapranov-Zelevinskĭ system for the primitive cohomology of an infinite series of complete intersection Calabi-Yau manifolds, whose dimension is the loop order minus one, we completely clarify the analytic structure of all banana integrals with arbitrary masses. In particular, we find that the leading logarithmic structure in the high energy regime, which corresponds to the point of maximal unipotent monodromy, is determined by a novel $\widehat{\Gamma}$-class evaluation in the ambient spaces of the mirror, while the imaginary part of the integral in this regime is determined by the $\widehat{\Gamma}$-class of the mirror Calabi-Yau manifold itself. We provide simple closed all loop formulas for the former as well as for the Frobenius $\kappa$-constants, which determine the behaviour of the integrals when the momentum square equals the sum of the masses squared, in terms of zeta values. We extend our previous work from three to four loops by providing for the latter case a complete set of (inhomogeneous) Picard-Fuchs differential equations for arbitrary masses. This allows to evaluate the banana integral in very short time to very high numerical precision for all values of the physical parameters. Using modular properties of the periods we determine the value of the maximal cut equal mass four-loop integral at the attractor points in terms of periods of modular weight two and four Hecke eigenforms and the quasiperiods of their meromorphic cousins.

KEYwords: Differential and Algebraic Geometry, Scattering Amplitudes

ArXiv EPrint: 2008.10574

\footnotetext{
${ }^{1}$ Corresponding author.
} 


\section{Contents}

1 Introduction 1

2 Banana Feynman integrals $\quad 4$

2.1 The $l$-loop banana integral and its geometrical realization 4

2.2 Bessel function representation of $l$-loop banana integrals $\quad 7$

2.3 The maximal cut integral for large momentum 8

3 The $l$-loop equal mass banana Feynman integral $\quad 9$

3.1 Inhomogeneous differential equation for the $l$-loop equal mass banana Feynman integral 9

3.2 Analytic properties of the l-loop equal mass banana graph Feynman integral 11

$\begin{array}{lll}3.3 & \text { Frobenius basis at the MUM point } & 12\end{array}$

3.4 Banana Feynman integral in terms of the MUM-Frobenius basis 13

$\begin{array}{ll}3.5 \text { Monodromy } & 15\end{array}$

$\begin{array}{lll}3.6 & \text { Special points } & 17\end{array}$

4 The $\widehat{\Gamma}$-class and zeta values at the point of maximal unipotent mon$\begin{array}{lr}\text { odromy } & 18\end{array}$

5 The $l$-loop non-equal mass banana Feynman integrals 26

5.1 Batyrev coordinates and the maximal cut integral 26

$\begin{array}{lll}5.2 & \text { Differential equations for the non-equal mass case } & 27\end{array}$

5.3 Linear combination for the non-equal mass Feynman integral 31

6 Conclusion and outlook $\quad 31$

A Derivation of the Bessel function representation 33

B Inhomogeneous differential equation for the four-loop case 34

$\begin{array}{ll}\text { C Pari/GP script for the equal mass integral } & 35\end{array}$

\section{Introduction}

Precision calculations of physical observables within a perturbative QFT usually require the evaluation of (many) Feynman integrals up to a certain loop order. This includes, perhaps most prominently, cross sections for high-energy collider experiments. In order to make the most out of an increasing amount of experimental data collected e.g. at the Large Hadron Collider, theoretical predictions need to be sharpened by computing higher 


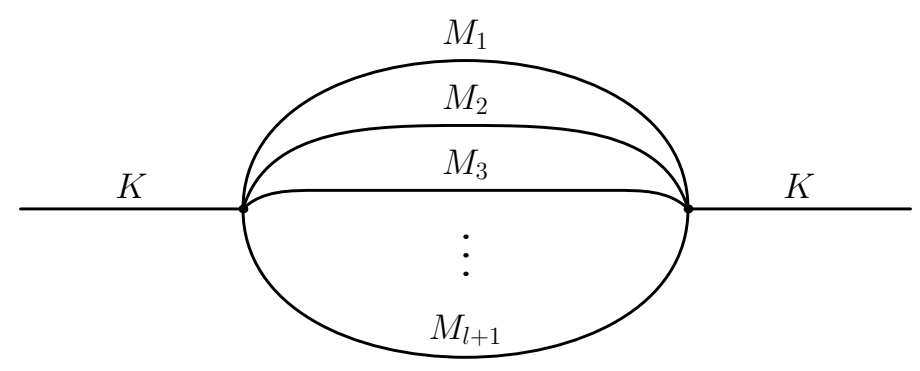

Figure 1. The $l$-loop banana diagram with external momentum $K$ and internal masses $M_{i}$.
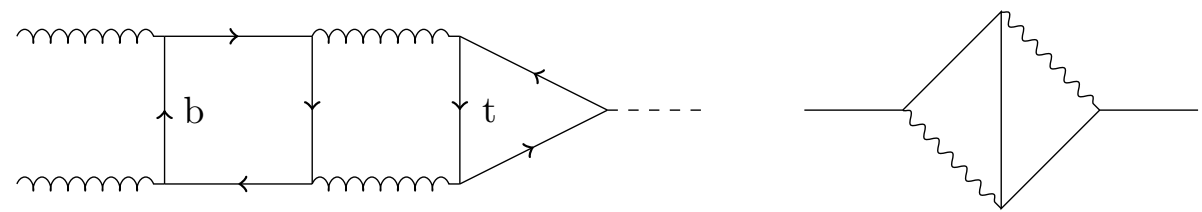

Figure 2. A three loop contribution to Higgs production via gluon fusion with a bottom and a top quark running in the loops (left panel). The scalar kite Feynman graph with two massless and three propapagators of equal mass (right panel).

loop orders in the perturbative expansion. This poses challenging computational problems that come along with a series of deep questions regarding the mathematical structures underlying multi-loop Feynman integrals.

A concrete problem of this kind is defined by the class of Feynman integrals associated with so-called banana graphs that will be considered in the present work (see figure 1). Part of their practical relevance comes from the fact that banana type graphs often appear as a subtopology of more complicated (more realistic) Feynman graphs, i.e., they are obtained by contracting a suitable subset of internal lines. To understand this, first note that, after suitable tensor and Dirac algebra manipulations in the numerator of a given Feynman integral (say involving fermion or gauge boson propagators), the problem can generically be reduced to the computation of a set of scalar Feynman integrals, possibly with nontrivial but scalar numerator (in momentum space). Now scalar Feynman integrals satisfy in dimensional regularization integration-by-parts identities $[1,2]^{1}$ (IBP) which allow to further reduce to a smaller (finite) number of integrals, commonly called master integrals of the respective problem. Typically the latter are Feynman integrals associated with subtopologies in the above sense and in this way banana type integrals ${ }^{2}$ frequently arise for instance as master integrals in two-loop electro-weak computations [6], in the two-loop Higgs + jet production cross section [7], in three-loop corrections to the $\rho$-parameter [8] or at four-loop order in the anomalous magnetic moment of the electron [9].

Another example is the so-called kite integral (see figure 2), which gives a contribution to the two-loop electron self-energy. The subtopologies in this example sit in the inhomogeneous term of its first order differential equation [10]. More generally, the kite family

\footnotetext{
${ }^{1}$ These identities are reviewed in for example [3-5].

${ }^{2}$ Here the banana type integrals may have propagator powers $\nu_{i}$ different from unity, in which case one assignes a Feynman graph with $\nu_{k}-1$ dots on the $k$ th propagator.
} 
with arbitrary powers of the respective propagators has a set of eight master integrals that satisfy a first-order Fuchsian differential system in the momentum parameter. Here two massive two-loop banana type integrals, once with primitive powers of the propagators and once with a propagator raised to the second power, form together with the two-loop tadpole integral $^{3}$ a closed differential subsystem [10]. ${ }^{4}$ Computational control over the subsectors may now be regarded as a key step for solving the full problem. In this spirit explicit analytic results for the three-loop non-equal mass banana Feynman integral are expected to form an important contribution to analytically computing the Higgs production cross section in QCD via gluon fusion with heavy quarks running in the loops, see figure $2 .{ }^{5}$

Higher loop banana integrals also constitute important case studies on the mathematical structure of multi-loop Feynman integrals and the development (or refinement) of appropriate computational tools. Often Feynman integrals give rise to interesting classes of special functions such as multiple polylogarithms. The latter, however, are not sufficient. In fact, the two-loop banana integral with non-zero masses is known to be the simplest Feynman integral that is not expressible in terms of multiple polylogarithms and it has spurred generalizations to elliptic multiple polylogarithms and iterated integrals of modular forms $[8,11,14,15]$. Nevertheless, the function theory for higher loop integrals is, especially for different non-zero masses, less understood and explicit results are scarce. The present work, building on [16], significantly improves this situation for the case of banana type integrals by employing suitable algebro-geometric techniques, well-known in the context of topological string theory on Calabi-Yau manifolds [17, 18]. Recall that Feynman integrals, more precisely their Laurent coefficients in the dimensional regularization parameter, are period integrals [19] in the sense of Kontsevich and Zagier [20]. As a function of their physical parameters (and possibly auxiliary deformation parameters), they satisfy Picard-Fuchs differential equations describing the variation of mixed Hodge structures [21, 22]. Especially, in combination with combinatoric techniques for appropriate toric varieties [23] these equations become a powerful approach to compute the banana Feynman integrals [16, 24]. This eventually allows for a complete determination of such a Feynman integral in terms of local Frobenius bases of solutions to the Picard-Fuchs differential ideal, which describes a Gelfand-Kapranov-Zelevinskĭ (GKZ) generalized hypergeometric system [25-27]. ${ }^{6}$ For the Feynman integral this is an inhomogeneous differential system, the inhomogeneity resulting from the fact that the integration domain has non-trivial boundary so the Feynman integral becomes a relative period. ${ }^{7}$ The homogeneous solutions of the system in turn describe period integrals of the same integrand over closed cycles (i.e. without boundary)

\footnotetext{
${ }^{3}$ Note that the only subtopology of the $l$-loop banana graph is a tadpole of $l$-loop bouqet topology.

${ }^{4}$ Also for the family of three-loop equal mass banana type integrals one finds $3+1$ master integrals, one of which is a tadpole contribution that can be recast to an inhomogeneity in the differential system of the remaining three integrals [11]. See also [12,13] for counts of master integrals for banana families.

${ }^{5}$ We thank Claude Duhr for pointing out to us the connection between the three-loop banana integrals and the gluon fusion process depicted in figure 2 .

${ }^{6}$ See $[28-32]$ for further applications of GKZ systems and $[33,34]$ for those of toric geometry to Feynman integrals.

${ }^{7}$ Here we refer to the integration domain $\sigma_{l}$ in the parametric represenation of the Feynman integral, see equations (2.1) and (2.2).
} 
in the cohomology of a family of Calabi-Yau $(l-1)$-folds, and the maximal cut integral of the Feynman graph turns out to be a special case thereof. ${ }^{8}$ In practice this means that the (full) Feynman integral will be a linear combination of the homogeneous solutions (a Frobenius basis for the closed periods) and a special inhomogeneous solution (regarded as a special relative period). ${ }^{9}$

The paper is organized as follows: in section 2 we recall the Feynman and Bessel representation of the banana integrals, comment on the underlying Calabi-Yau families and compute the maximal cut integral. In section 3 we re-derive the equal-mass case differential equation, find Frobenius solutions in the large momentum regime, express the Feynman integral in terms of the latter and discuss monodromies and singularities. The resulting expansion is compared to the $\widehat{\Gamma}$-conjecture for mirror Calabi-Yau geometries in section 4. Picard-Fuchs operators, multi-variate Frobenius bases and the correct choice of linear combination for the banana integral in the non-equal mass case are provided in section 5. Section 6 contains conclusions and open problems. Appendix A concerns the Bessel representation of banana integrals and appendix $\mathrm{B}$ deals with an explicit inhomogeneous differential equation for the four-loop generic mass case. The third appendix $\mathrm{C}$ explains our Pari/GP script BananaAmplitude.gp which computes the equal mass banana integral.

\section{Banana Feynman integrals}

In this section we introduce the main object we focus on in this paper, namely the l-loop banana Feynman integral and make first comments on the underlying geometry. Moreover, we give a representation of the Feynman integral in terms of Bessel functions valid for small momenta. In the large momenta regime we calculate the maximal cut integral.

\subsection{The $l$-loop banana integral and its geometrical realization}

The $l$-loop banana Feynman graph is shown in figure 1. By means of standard textbook Feynman rules one can write down the Feynman integral associated to each such banana graph. For our purpose it is, however, more convenient to use the Symanzik parametrization of Feynman integrals (see e. g. [37] for an introduction). In two dimensions the $l$-loop Feynman integral thus reads ${ }^{10}$

$$
\mathcal{F}_{\sigma_{l}}\left(t, \xi_{i}\right)=\int_{\sigma_{l}} \frac{\mu_{l}}{P_{l}\left(t, \xi_{i} ; x\right)}=\int_{\sigma_{l}} \frac{\mu_{l}}{\left(t-\left(\sum_{i=1}^{l+1} \xi_{i}^{2} x_{i}\right)\left(\sum_{i=1}^{l+1} x_{i}^{-1}\right)\right) \prod_{i=1}^{l+1} x_{i}} .
$$

Here the edge variables $x_{i}$ form a set of homogeneous coordinates for the projective space $\mathbb{P}^{l}$ and the $l$ real dimensional integration domain $\sigma_{l}$ is defined as

$$
\sigma_{l}=\left\{\left[x_{1}: \ldots: x_{l+1}\right] \in \mathbb{P}^{l} \mid x_{i} \in \mathbb{R}_{\geq 0} \text { for all } 1 \leq i \leq l+1\right\},
$$

\footnotetext{
${ }^{8}$ Also see $[35,36]$ for a connection between the maximal cut integral and homogeneous differential equations.

${ }^{9}$ For simplicity we will sometimes just speak of a Frobenius basis, which then includes the special solution.

${ }^{10} \mathrm{As}$ a consequence of the $i \epsilon$ prescription one has to shift $t \rightarrow t+i \epsilon$ and consider the limit $\epsilon \rightarrow 0$.
} 
while the holomorphic $l$-measure $\mu_{l}$ is defined by

$$
\mu_{l}=\sum_{k=1}^{l+1}(-1)^{k+1} x_{k} \mathrm{~d} x_{1} \wedge \ldots \wedge \widehat{\mathrm{d} x_{k}} \wedge \ldots \wedge \mathrm{d} x_{l+1} .
$$

As usual, the hat indicates the omission of one differential. In writing (2.1) we have also introduced dimensionless kinematical parameters,

$$
t=\frac{K^{2}}{\mu^{2}}, \quad \text { and } \quad \xi_{i}=\frac{M_{i}}{\mu} \quad(i=1, \ldots l+1),
$$

where $K$ is the external momentum, the $M_{i}$ are the $l+1$ propagator masses and $\mu$ is an arbitrary scale.

By definition a Calabi-Yau $(l-1)$-fold is a complex Kähler manifold, meaning that it has a Kähler form $\omega$ of Hodge type $(1,1)$, which in addition has a vanishing first Chern class $c_{1}$ and a unique nowhere vanishing holomorphic $(l-1,0)$-form $\Omega_{l-1}$. A standard way to construct such manifolds is to embed them as hypersurfaces or complete intersections in complex Kähler manifolds with positive first Chern class, in particular in Fano varieties, so that the Chern class of the embedded variety vanishes. ${ }^{11}$ For example an elliptic curve, a Calabi-Yau manifold of complex dimension one, can be defined in the simplest realization as the vanishing locus of a cubic hypersurface in $\mathbb{P}^{2}$. A key observation is that the integral (2.1) can be understood as a (relative-) period integral for a smooth family of $(l-1)$-dimensional Calabi-Yau hypersurfaces $M_{l-1}^{l^{2}}$ in a Fano ambient space, which generically have $\operatorname{dim} H^{1}\left(M_{l-1}^{l^{2}}, T M_{l-1}\right)=h^{l-2,1}=l^{2}$ complex structure deformations. This generalizes the elliptic functions that arise for the two-loop case as periods over the holomorphic one-form of the elliptic curve to the Calabi-Yau period integrals over the unique holomorphic form $\Omega_{l-1}$ in the higher loop case. The relevant families of higher dimensional Calabi-Yau manifolds are defined as vanishing locus

$$
M_{l-1}^{l^{2}}=\left\{P_{\Delta_{l}}(\underline{y})=0 \mid \underline{y} \in \mathbb{P}_{\hat{\Delta}_{l}}\right\}
$$

of the Laurent polynomial $P_{\Delta_{l}}=P_{l}\left(t, \xi_{i} ; x\right) / \prod_{i=1}^{l+1} x_{i}$ in the coordinate ring of the toric Fano ambient space $\mathbb{P}_{\hat{\Delta}_{l}}$, where $\Delta_{l}$ is the $l$-dimensional reflexive Newton polytope of the polynomial $P_{\Delta_{l}}$ and $\hat{\Delta}_{l}$ is its dual. A complete description of the reflexive pair of lattice polyhedra $\left(\Delta_{l}, \hat{\Delta}_{l}\right)$ and the associated Fano- $\left(\mathbb{P}_{\Delta_{l}}, \mathbb{P}_{\hat{\Delta}_{l}}\right)$ and Calabi-Yau (mirror) geometries $\left(M_{l-1}, \widehat{M_{l-1}}\right)$ can be found in [16], whose notation we follow. By Batyrev's mirror construction [38] the mirror $W_{l-1}$ is given as in (2.5) but with the rôles of $\Delta_{l}$ and $\hat{\Delta}_{l}$ exchanged.

We note that a single residuum integral $\Omega_{l-1}=\operatorname{Res}_{P_{l}\left(t, \xi_{i} ; x\right)=0}\left(\mu_{l} / P_{l}\left(t, \xi_{i} ; x\right)\right)$ yields an expression for the holomorphic $(l-1,0)$-form on the Calabi-Yau manifolds $M_{l-1}^{l^{2}}$. This was used in [16] to derive from the Gelfand-Kapranov-Zelevinskı̌ (GKZ) differential system up to three loops the differential D-module describing those geometrical integrals over $\Omega_{l-1}$ that yield the physical Feynman integral (2.1) in all regions of their physical parameter space in $t$ and $\xi_{i}$ for $i=1, \ldots, l+1$.

\footnotetext{
${ }^{11}$ See section 4 for the concept of Chern classes and (4.13) for an explicit check that the first Chern classes $c_{1}$ of the embedded Calabi-Yau manifolds vanish.
} 
In the present work we extend the program of [16] to banana Feynman diagrams of all loop orders $l$. As in [16] a key technical step is to reduce the solutions of the $l^{2}$ parameter GKZ system to the subset of solutions that describe the physical periods in the $l+1$ physical parameters, which we achieved starting from the GKZ system of $M_{l-1}^{l^{2}}$. Even though the full differential D-module $\mathcal{D}^{l}$ is lengthy to write down and will be made explicit only up to $l=4$, we can provide a complete analytic description for the integral for the $l$-loop banana graph $\mathcal{F}_{\sigma_{l}}\left(t, \xi_{i}\right)$ in all regions of the moduli space. The latter is based on the identification of certain universal operators in $\mathcal{D}^{l}$ and systematic analytic continuation formulas valid for all $l$, which involves a systematic occurrence of products of zeta values with highest transcendentality $l$.

An alternative geometric description. It is natural to expect that the products of zeta values are related to the $\widehat{\Gamma}$-class of the mirror Fano threefold $\mathbb{P}_{\hat{\Delta}_{l}}$. Due to the very high co-dimension of the Kähler subslice mirror dual to the $(l+1)$-dimensional physical slice of (complex structure) parameters of $M_{l-1}^{l^{2}}$, confirming this is an increasingly complicated task. However, Matt Kerr pointed out to us that for the Fano variety which is associated to the equal mass three-loop banana diagram there is a realization of its Hodge structure that is an alternative to the redundantly parametrized one of $\mathbb{P}_{\hat{\Delta}_{l}}$, namely this alternative is simply a degree $(1,1,1,1)$ hypersurface in $\left(\mathbb{P}^{1}\right)^{4}$. This key observation was made by identifying the holomorphic solution associated to the differential system of this integral with the one that appears in an example ${ }^{12}$ in the list [39] and has the same description. This suggests that the relevant physical subslices in the series of the Calabi-Yau manifolds $M_{l-1}^{l^{2}}(2.5)$ are dual to complete intersections of two degree $(1, \ldots, 1)$ constraints in $\left(\mathbb{P}^{1}\right)^{l+1}$. The GKZ systems of complete intersections have been studied in [17] under the aspect of mirror symmetry. So a good model for the Calabi-Yau $(l-1)$-fold $W_{l-1}$ is the complete intersection of two degree $(1, \ldots, 1)$ constraints in $\left(\mathbb{P}^{1}\right)^{l+1}$ that reads in the notation of $[17]$

$$
\left.\left.W_{l-1}^{l}=\left(\begin{array}{c||c|c}
\mathbb{P}_{1}^{1} & 1 & 1 \\
\vdots & \vdots & \vdots \\
\mathbb{P}_{l+1}^{1} & 1 & 1
\end{array}\right\} l+1\right) \subset\left(\begin{array}{c||c}
\mathbb{P}_{1}^{1} & 1 \\
\vdots & \vdots \\
\mathbb{P}_{l+1}^{1} & 1
\end{array}\right\} l+1\right)=F_{l},
$$

which is here suitably embedded ${ }^{13}$ in the Fano $l$-fold $F_{l}$. According to [17] the mirror manifold $M_{l-1}^{l+1}$ is given by a resolved quotient of (2.6) $M_{l-1}=\widehat{W_{l-1} / G}$ and the period geometry of $M_{l-1}^{l+1}$ is defined by the invariant periods of $W_{l-1} / G$ depending on the $G$-invariant $l$-dimensional deformation space. This construction is a special case of the construction of Batyrev and Borisov [40].

This suggests that in the high energy regime the physical mass and momentum parameters should be identified with the complexified Kähler parameters

$$
\mathfrak{t}^{k}=\frac{1}{2 \pi i} \int_{\mathbb{P}_{k}^{1}}(i \omega-b),
$$

\footnotetext{
${ }^{12}$ This example is given in the link http://coates.ma.ic.ac.uk/fanosearch/?page_id=277\#4-1.

${ }^{13}$ The lower index on the manifolds (apart from $\mathbb{P}_{k}^{1}$ of course) indicates their complex dimension in terms of the number of loops $l$ of the Feyman diagram.
} 
which control the area $A_{k}=\frac{1}{2 \pi} \int_{\mathbb{P}_{k}^{1}} \omega$ of the $k$ th $\mathbb{P}^{1}$ in $(2.6)$, via

$$
\mathfrak{t}^{k} \simeq \frac{1}{2 \pi i} \log \left(\frac{M_{k}^{2}}{K^{2}}\right)=\frac{1}{2 \pi i} \log \left(z_{k}\right) \quad \text { for } k=1, \ldots, l+1 .
$$

Here $\omega$ is the Kähler $(1,1)$-form on $W_{l-1}$ that exists by definition on any Calabi-Yau manifold, see e.g. [18] for a review. The complexification of the area in (2.7) is by the expectation value of the Neveu-Schwarz $(1,1)$-form field $b$ [18]. On the other side the $z_{k}$ are the canonical complex structure variables of $M_{l-1}$, chosen so [17] that the point of maximal unipotent monodromy of the Picard-Fuchs (or Gauss-Manin) system of $M_{l-1}$ is at $z_{k}=0$. The precise relation will be given in (4.4). As explained in [18] the complexified Kähler parameters are identified under mirror symmetry with the complex deformation parameters of $M_{l-1}$, and thus with the parameters of the integral (2.1). If the identification (2.8) is true we expect that the large energy behaviour of the Feynman integral is exactly determined by the quantum cohomology of $W_{l-1} \subset F_{l}$ in the large volume limit of the geometry. In particular, if this beautiful picture holds we can infer the entire leading logarithmic structure of the Feynman integral from the central charge of the corresponding object in the derived category of coherent sheaves, which can be described by the $\widehat{\Gamma}$-class conjecture in terms of the topological data of $W_{l-1}$ and $F_{l}$ and which can be easily controlled for all loop orders $l$. This will be made explicit in section 4 .

Comparing the geometric descriptions. We establish the equivalence of the above two geometric descriptions by first deriving the Picard-Fuchs equations of the Feynman graph geometry (2.1) and (2.5) as a Calabi-Yau hypersurface in a toric variety (description 1), reducing its GKZ system to the physical parameters and finding the corresponding solutions, as will be done in sections 3 and 5 . These data can then be compared to the GKZ system for the complete intersection (2.6) (description 2) and its solutions given in [17] after a change of variables, as will be discussed in section 4 . Note, however, that the GKZ systems given in [17] in generality do not yield immediately the complete PicardFuchs differential ideal for closed Calabi-Yau periods (which are sufficient to describe the maximal cut integral). We solved this problem for the homogeneous system for the CalabiYau periods and the extension to the inhomogeneous system for the three-loop graph in [16] and for the four-loop graph in this work. In the general loop case we can check that the holomorphic solutions (2.13) and (4.9), which can in both geometries be derived from a simple residuum integral near the point of maximal unipotent monodromy, indeed agree upon a suitable identification of the variables.

\subsection{Bessel function representation of $l$-loop banana integrals}

Besides the parametric representation (2.1), we also recall a representation of the Feynman integral in terms of an integral over Bessel functions, which in its regime of validity,

$$
t<\left(\sum_{i=1}^{l+1} \xi_{i}\right)^{2}
$$


is well suited for numerical evaluation. Relegating a short derivation to appendix A, the Feynman integral (2.1) can be rewritten as

$$
\mathcal{F}_{\sigma_{l}}=2^{l} \int_{0}^{\infty} z I_{0}(\sqrt{t} z) \prod_{i=1}^{l+1} K_{0}\left(\xi_{i} z\right) \mathrm{d} z .
$$

In particular, in the equal mass case the expression (2.10) contains the $(l+1)$ th symmetric power of the Bessel function $K_{0}$.

As a side remark, in the on-shell case (defined via $t=\xi_{i}=1$ for all $i$ ) the integral (2.10) becomes a special instance of a Bessel moment. Bessel moments differ in their powers of $z, I_{0}(z)$ and $K_{0}(z)$ in the integrand. The massive vacuum banana integrals also yield Bessel moments [41, 42]. Such Bessel moments have also caught the interest of number theorists, one reason being that they, in some cases, evaluate to critical values of L-series of certain modular forms (as briefly reviewed in subsection 3.6). They satisfy many interesting relations [43-46] and are closely related to L-functions built from symmetric power moments of Kloosterman sums [47-49].

\subsection{The maximal cut integral for large momentum}

One goal of the paper is to analyze the l-loop banana graph in the regime $t>\left(\sum_{i=1}^{l+1} \xi_{i}\right)^{2}$ where the expression (2.10) becomes invalid. It turns out that there is an elegant expression for the so-called maximal cut integral associated with the banana graph, which still contains substantial information about the full Feynman integral $\mathcal{F}_{\sigma_{l}}$.

The maximal cut integral is obtained by replacing all ${ }^{14}$ propagators by delta functions. As derived in [24] there is again a parametric representation of the maximal cut integral in terms of the Symanzik polynomials. To get the maximal cut integral one simply changes the integration range from the simplex $\sigma_{l}$ to the $l$-torus $T^{l}$. So for the banana integrals we obtain

$$
\mathcal{F}_{T^{l}}\left(t, \xi_{i}\right)=\int_{T^{l}} \frac{\mu_{l}}{\left(t-\left(\sum_{i=1}^{l+1} \xi_{i}^{2} x_{i}\right)\left(\sum_{i=1}^{l+1} x_{i}^{-1}\right)\right) \prod_{i=1}^{l+1} x_{i}} .
$$

Now, for large momenta $t$, the maximal cut integral $\mathcal{F}_{T^{l}}$ can be obtained explicitly by a simple residue calculation. Introduce the variable $s=1 / t$, then for small $s$ subsequent geometric series and multinomial expansion yields

$$
\begin{aligned}
\mathcal{F}_{T^{l}}\left(t, \xi_{i}\right)= & \int_{T^{l}} \frac{s}{1-s\left(\sum_{i=1}^{l+1} \xi_{i}^{2} x_{i}\right)\left(\sum_{i=1}^{l+1} x_{i}^{-1}\right)} \frac{\mu_{l}}{\prod_{i=1}^{l+1} x_{i}} \\
= & \int_{T^{l}} \sum_{n=0}^{\infty} s^{n+1}\left(\sum_{|k|=n}\left(\begin{array}{c}
n \\
k_{1}, \ldots, k_{l+1}
\end{array}\right) \prod_{i=1}^{l+1}\left(\xi_{i}^{2} x_{i}\right)^{k_{i}}\right) \\
& \times\left(\sum_{|\tilde{k}|=n}\left(\begin{array}{c}
n \\
\tilde{k}_{1}, \ldots, \tilde{k}_{l+1}
\end{array}\right) \prod_{i=1}^{l+1} x_{i}^{-\tilde{k}_{i}}\right) \frac{\mu_{l}}{\prod_{i=1}^{l+1} x_{i}} \\
= & (2 \pi i)^{l} \sum_{n=0}^{\infty} s^{n+1} \sum_{|k|=n}\left(\begin{array}{c}
n \\
k_{1}, \ldots, k_{l+1}
\end{array}\right)^{2} \prod_{i=1}^{l+1} \xi_{i}^{2 k_{i}}
\end{aligned}
$$

\footnotetext{
${ }^{14}$ One can also consider non-maximal cuts where only some propagators are replaced by delta functions. For our purpose these cut integrals are not relevant.
} 
Here we used the short hand notation $|k|=\sum_{i=1}^{l+1} k_{i}$ and evaluated a multidimensional residue in the last step. So up to normalization the maximal cut integral is for large momentum given by

$$
\varpi_{0}\left(s, \xi_{i}\right)=\sum_{n=0}^{\infty} s^{n+1} \sum_{|k|=n}\left(\begin{array}{c}
n \\
k_{1}, \ldots, k_{l+1}
\end{array}\right)^{2} \prod_{i=1}^{l+1} \xi_{i}^{2 k_{i}} .
$$

When expressed in terms of the variable $t=1 / s$, one recovers [24, eq. (123)].

\section{The $l$-loop equal mass banana Feynman integral}

In this section we focus on the equal mass case, i.e. $\xi_{i}=1$ for $i=1, \ldots l+1$. We first derive an inhomogeneous differential equation for the equal mass Feynman integral. This equation is already known in the literature $[22,50]$, however, the derivation presented here is somewhat different. It is based on the observation that the maximal cut integral in the large momentum regime is given by the double Borel sum of a certain function for which one can easily construct an operator that annihilates it. This relation makes the computation of the desired differential equation conceptually clear and easy. For each $l$ the differential equation thus obtained is related to the one in [22] by simply transforming it to the small momentum regime. We subsequently explain the analytic properties of its solutions and compare to the actual Feynman integral. Coefficients relating local solutions in the large momentum regime to the Feynman integral are given. A conjectural relation for these coefficients to the so-called $\widehat{\Gamma}$-class is proposed. Moreover, these coefficients are linked to the Frobenius $\kappa$-constants as we explain before ending the section with some remarks about special points of the Feynman integral.

\subsection{Inhomogeneous differential equation for the l-loop equal mass banana Feynman integral}

In this subsection we give an elegant description for the $l$-loop banana Feynman integral which easily leads to its inhomogeneous differential equation.

First consider the maximal cut integral $\mathcal{F}_{T^{l}}$ for large momenta $t$, i.e. near $s=0$, as given in eq. (2.13). In the equal mass case, i.e. $\xi_{i}=1$, the expression $\varpi_{0} / s$ can be seen as the double Borel sum ${ }^{15}$ of the $(l+1)$ th symmetric power of the series

$$
\sum_{k=0}^{\infty} \frac{1}{(k !)^{2}} x^{k}=I_{0}(2 \sqrt{x}) \text {. }
$$

Note that the double Borel sum resides, simply speaking, in the additional factor of $(n !)^{2}$ in the coefficients of (2.13), relative to those of the symmetric power of the Bessel function.

Hence, the differential equation annihilating the maximal cut integral (2.13) can be derived by three steps: first calculate the differential equation for the $(l+1)$ th symmetric power of (3.1). Second, by a simple analysis of the (double) Borel sum we can infer

\footnotetext{
${ }^{15} \mathrm{Or}$ in other words, the $(l+1)$ th symmetric power of $I_{0}$ is the Borel transform of the Borel transform of $\varpi / s$.
} 
the differential operator of the function $\varpi_{0} / s$ from the operator of the symmetric power. Third, the additional factor of $s$ is commuted into the differential operator to obtain the Picard-Fuchs equation for $\varpi_{0}$.

Step 1. The function $I_{0}(2 \sqrt{x})$ is annihilated by the operator

$$
\mathcal{D}=\theta^{2}-x
$$

with the logarithmic derivative $\theta=x \partial_{x}$. For the $(l+1)$ th symmetric power of this function we use a result from $[51,52]$ :

Lemma. Let $\mathcal{D}=\theta^{2}+a(x) \theta+b(x)$ be a linear differential operator whose coefficients $a(x)$ and $b(x)$ are rational functions. Let $\mathcal{L}_{0}=1, \mathcal{L}_{1}=\theta$ and for $k=1,2, \ldots, n$ define the operator $\mathcal{L}_{k+1}$ by

$$
\mathcal{L}_{k+1}=(\theta+k a(x)) \mathcal{L}_{k}+k b(x)(n-k+1) \mathcal{L}_{k-1}
$$

Then the symmetric power $y^{n}$ of any solution to $\mathcal{D} y=0$ is annihilated by $\mathcal{L}_{n+1}$.

In the case at hand we have $a(x)=0$ and $b(x)=-x$ while $n=l+1$.

Step 2. For the Borel summation we notice the following properties: given a power series $\Psi(x)=\sum_{n} a_{n} x^{n}$, its Borel transform is defined by $\mathcal{B} \Psi(z)=\sum_{n} a_{n} \frac{z^{n}}{n !}$. The original series $\Psi(x)$ is obtained from the Borel transform $\mathcal{B} \Psi(z)$ by the back-transformation

$$
\Psi(x)=\int_{0}^{\infty} \mathrm{e}^{-z} \mathcal{B} \Psi(z x) \mathrm{d} z,
$$

which is similar to a Laplace transformation, the right hand side now being referred to as the Borel sum of $\Psi(x)$. Given a differential operator annihilating the Borel transform $\mathcal{B} \Psi(z)$ we can infer the corresponding operator annihilating the original function $\Psi(x)$, simply by analyzing the relation (3.4). The rules

$$
\begin{aligned}
& \theta_{z}^{n} \mathcal{B} \Psi(z) \longrightarrow \theta_{x}^{n} \Psi(x) \\
& z^{n} \mathcal{B} \Psi(z) \longrightarrow\left(x\left(1+\theta_{x}\right)\right)^{n} \Psi(x)=\theta_{x}\left(x^{n} \prod_{k=1}^{n-1}\left(\theta_{x}+k\right)\right) \Psi(x)
\end{aligned}
$$

are useful in this respect, where $\theta_{x, z}$ are the logarithmic derivatives in $x$ and $z$, respectively. After each back-transformation, i.e., application of the rules (3.5), we can factor out a logarithmic derivative $\theta_{x}$ since it turns out that the degree of the differential operator for the Borel transform of $\varpi_{0} / s$ is increased by one compared to the original function $\varpi_{0} / s$.

Step 3. Finally, we remark that given a function $f(x)$ and an operator $\mathcal{D}$ with $\mathcal{D} f=0$ the function $\phi(x)=x f(x)$ is annihilated by the operator $\tilde{\mathcal{D}}$, which is obtained from $\mathcal{D}$ by replacing $\theta \rightarrow \theta-1$.

Putting all together we obtain the homogeneous degree $l$ operator $\mathcal{L}_{l}$ annihilating the equal mass maximal cut integral $\mathcal{F}_{T^{l}}$. It turns out that this operator is of Fuchsian type 


\begin{tabular}{|cl|}
\hline \#Loops $l$ & Differential operator $\mathcal{L}_{l}$ \\
\hline 1 & $1-2 s+(-1+4 s) \theta$ \\
2 & $1-3 s+(-2+10 s) \theta+(-1+s)(-1+9 s) \theta^{2}$ \\
3 & $1-4 s+(-3+18 s) \theta+(3-30 s) \theta^{2}-(-1+4 s)(-1+16 s) \theta^{3}$ \\
4 & $1-5 s+(-4+28 s) \theta+\left(6-63 s+26 s^{2}-225 s^{3}\right) \theta^{2}+\left(-4+70 s-450 s^{3}\right) \theta^{3}$ \\
& $-(-1+s)(-1+9 s)(-1+25 s) \theta^{4}$ \\
5 & $1-6 s+(-5+40 s) \theta+\left(10-112 s+1152 s^{3}\right) \theta^{2}$ \\
& $+\left(-10+168 s-236 s^{2}+4608 s^{3}\right) \theta^{3}+\left(5-140 s+5760 s^{3}\right) \theta^{4}$ \\
& $+(-1+4 s)(-1+16 s)(-1+36 s) \theta^{5}$ \\
\hline
\end{tabular}

Table 1. Homogeneous differential operators for maximal cut integrals.

for any $l$. Using a computer algebra program such as Mathematica it is not hard to write a small program ${ }^{16}$ to generate the differential operators. The first few are listed in table 1.

For the full equal mass banana Feynman integral $\mathcal{F}_{\sigma_{l}}$ we have to extend these differential equations to inhomogeneous ones. By numerical evaluation of the integral $\mathcal{L}_{l} \mathcal{F}_{\sigma_{l}}$ one finds for the inhomogeneity

$$
\mathcal{L}_{l} \mathcal{F}_{\sigma_{l}}(s, 1)=S_{l}:=-(l+1) ! s
$$

\subsection{Analytic properties of the $l$-loop equal mass banana graph Feynman inte- gral}

In this subsection we study the analytic properties of the Frobenius basis corresponding to the (in-)homogeneous differential equaion (3.6) derived in the previous subsection. These properties partially descend to the actual Feynman integral, which is given by an appropriate linear combination. The coefficients of this linear combination will be computed in the next subsection.

We reserve the indices $k=0, \ldots, l-1$ to the homogeneous solutions of $\mathcal{L}_{l} \varpi_{k}=0$, while the index $k=l$ refers to the special solution of the inhomogeneous equation $\mathcal{L}_{l} \varpi_{l}=S_{l}$. In this notation the Feynman integral $\mathcal{F}_{\sigma_{l}}$ is a linear combination of the $\varpi_{k}$ with a non-zero contribution of the special solution $\varpi_{l}$. On the other hand, the maximal cut $\mathcal{F}_{T^{l}}$ of the Feynman integral only involves the homogeneous solutions $\varpi_{k}$ with $k=0, \ldots, l-1$.

First, we discuss the singular points of the differential equation. At $s=1 / t=0$ we have a point of maximal unipotent monodromy, in short a MUM point. This means that the local exponents (i.e. the roots of the indicial equation) of $\mathcal{L}_{l} \varpi_{k}=0$ are all equal. In the case at hand they are all equal to one, which can be derived from the fact that $\mathcal{L}_{l}=(1-\theta)^{l}+\mathcal{O}(s)$. Moreover, in the $s$ coordinate the singular loci are the roots of the

\footnotetext{
${ }^{16}$ On the webpage http://www.th.physik.uni-bonn.de/Groups/Klemm/data.php we upload a small Mathematica file including a program to generate these operators. They are normalized such that they start with one, i.e. if we set $\theta$ and $z$ to zero the operator $\mathcal{L}_{l}$ gives unity.
} 


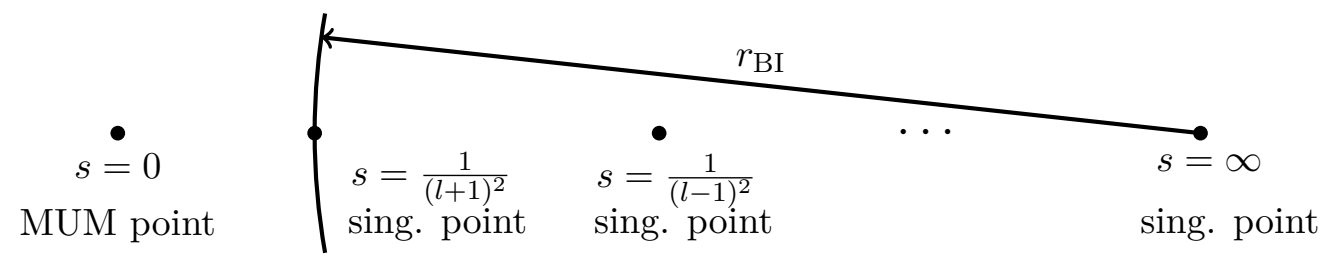

Figure 3. Singularities of the Fuchsian operator $\mathcal{L}_{l}$. The radius of convergence of the Bessel integral representation $(2.10)$ of $\mathcal{F}_{\sigma_{l}}$ is denoted by $r_{\mathrm{BI}}$.

discriminant $\Delta\left(\mathcal{L}_{l}\right)$, given by

$$
\Delta\left(\mathcal{L}_{l}\right)=s \prod_{j=0}^{\left\lceil\frac{l-1}{2}\right\rceil}\left(1-s(l+1-2 j)^{2}\right) .
$$

So in general, we have a moduli space

$$
\mathbb{P}^{1} \backslash\left(\bigcup_{j=0}^{\left\lceil\frac{l-1}{2}\right\rceil}\left\{\frac{1}{(l+1-2 j)^{2}}\right\} \cup\{0, \infty\}\right) .
$$

The actual Feynman integral $\mathcal{F}_{\sigma_{l}}$ is not singular at all of these points. From the Bessel function representation $(2.10)$ of $\mathcal{F}_{\sigma_{l}}$, valid in a neighbourhood of the point $s=\infty$ (i.e. $t=0)$ we know that it converges for $|s|>\frac{1}{(l+1)^{2}}$. In particular, this implies that the integral can only be singular at $s=0$ and $s=\frac{1}{(l+1)^{2}}$. This is also expected from the optical theorem since the latter point is a threshold for the $l+1$ particles in the loops (all of which have unit mass). At the other singular points of $\mathcal{L}_{l}$ the Feynman integral stays regular. In figure 3 this behavior is shown.

\subsection{Frobenius basis at the MUM point}

Around the MUM point the Frobenius basis takes a particularly nice form. The holomorphic solution $\varpi_{0}$ is given by $(2.13)$ at $\xi_{i}=1$ for $i=1, \ldots, l+1$. The other solutions can be chosen as ${ }^{17}$

$$
\varpi_{k}=\sum_{j=0}^{k}\left(\begin{array}{l}
k \\
j
\end{array}\right) \log (s)^{j} \Sigma_{k-j} \quad \text { for } k=1, \ldots, l-1,
$$

where $\Sigma_{0}=\varpi_{0}=s+\mathcal{O}\left(s^{2}\right)$ and the power series $\Sigma_{k}$ are determined by the operator $\mathcal{L}_{l}$ and the condition that they start as $\Sigma_{k}=\mathcal{O}\left(s^{2}\right)$ for $k \geq 1$. For example, the four-loop operator $\mathcal{L}_{4}$ has

$$
\begin{aligned}
& \varpi_{0}=s+5 s^{2}++45 s^{3}+545 s^{4}+7885 s^{5}+\cdots \\
& \Sigma_{1}=8 s^{2}+100 s^{3}+\frac{4148}{3} s^{4}+\frac{64198}{3} s^{5}+\cdots \\
& \Sigma_{2}=2 s^{2}+\frac{197}{2} s^{3}+\frac{33637}{18} s^{4}+\frac{2402477}{72} s^{5}+\cdots \\
& \Sigma_{3}=-12 s^{2}-\frac{267}{2} s^{3}-\frac{19295}{18} s^{4}-\frac{933155}{144} s^{5}+\cdots .
\end{aligned}
$$

\footnotetext{
${ }^{17}$ Note that the dependence on the loop order $l$ is kept implicit in our notation for $\varpi_{k}$ and $\Sigma_{k}$.
} 
The special solution $\varpi_{l}$ has one more logarithm and can be chosen as

$$
\varpi_{l}=\sum_{j=0}^{l}\left(\begin{array}{l}
l \\
j
\end{array}\right) \log (s)^{j} \Sigma_{l-j},
$$

which, after multiplication with the constant $(-1)^{l+1}(l+1)$, satisfies (3.6). Again for the four-loop example we find

$$
\Sigma_{4}=1830 s^{3}+\frac{112720}{3} s^{4}+\frac{47200115}{72} s^{5}+\cdots
$$

in the special solution $\varpi_{4}$.

The power series $\Sigma_{k}$ can also be obtained from a generating function approach starting from the holomorphic solution $\varpi_{0}$. To this end rewrite (2.13) in the form

$$
\varpi_{0}=\sum_{k_{1}, \ldots, k_{l+1} \geq 0}\left(\begin{array}{c}
|k| \\
k_{1}, \ldots, k_{l+1}
\end{array}\right)^{2} s^{|k|+1},
$$

where the summation is over all non-negative integers $k_{1}, \ldots, k_{l+1}$. Introduce formal parameters $\epsilon_{i}$ by replacing $k_{i} \rightarrow k_{i}+\epsilon_{i}$ in (3.13). Taking derivatives with respect to these parameters and subsequently putting the parameters to zero yields other Frobenius solutions ${ }^{18}$

$$
\left.\sum_{\left\{i_{1}, \ldots, i_{k}\right\} \in T_{k}^{(l+1)}} \frac{\partial^{k}}{\partial \epsilon_{i_{1}} \cdots \partial \epsilon_{i_{k}}} \varpi_{0}\left(\epsilon_{i}\right)\right|_{\text {all } \epsilon_{i}=0} \quad \text { for } k=1, \ldots, l,
$$

where $T_{k}^{(l+1)}$ denotes the set of all subsets of length $k$ of the set $T^{(l+1)}=\{1, \ldots, l+1\}$.

\subsection{Banana Feynman integral in terms of the MUM-Frobenius basis}

Recall that in a local Frobenius basis (for us the region around the MUM point is most interesting) the $l$-loop banana Feynman integral is given by a linear combination

$$
\mathcal{F}_{\sigma_{l}}=\sum_{k=0}^{l} \lambda_{k}^{(l)} \varpi_{k}
$$

In the following we explain how to obtain the coefficients $\lambda_{k}^{(l)}$.

The equal mass banana Feynman integrals $\mathcal{F}_{\sigma_{l}}$ can be evaluated numerically for fixed value of the variable $s$. For $l=2,3,4$ this can directly be done with the form given in (2.1), say using a numerical integration routine of Mathematica or Pari. Unfortunately, the multidimensional numerical integration gets too cumbersome for higher loop integrals due to the increase of the numerical error. On the other side, numerical integration is less problematic for the integral over Bessel functions (2.10), which can almost be computed for any loop order with any desired precision. However, around the MUM point $s=0$ the

\footnotetext{
${ }^{18}$ The functions produced here may be linear combinations of the previously considered solutions to the homogeneous differential equation. In other words, by forming appropriate linear combinations of the expressions in (3.14) one in turn obtains the $\Sigma_{k}$ as defined before.
} 


\begin{tabular}{|c|c|c|c|c|c|c|}
\hline$l$ & $\varpi_{0}$ & $\varpi_{1}$ & $\varpi_{2}$ & $\varpi_{3}$ & $\varpi_{4}$ & $\varpi_{5}$ \\
\hline 1 & $-2 \pi i$ & & & & & \\
\hline 2 & $18 \zeta(2)$ & $6 \pi i$ & & & & \\
\hline 3 & $-16 \zeta(3)+24 i \pi \zeta(2)$ & $-72 \zeta(2)$ & $-12 \pi i$ & & & \\
\hline 4 & $-450 \zeta(4)-80 i \pi \zeta(3)$ & $80 \zeta(3)-120 \pi i \zeta(2)$ & $180 \zeta(2)$ & $20 \pi i$ & & \\
\hline 5 & $\begin{array}{c}-288 \zeta(5)+1440 \zeta(2) \zeta(3) \\
-540 i \pi \zeta(4)\end{array}$ & $\begin{array}{c}2700 \zeta(4) \\
+480 i \pi \zeta(3)\end{array}$ & $\begin{array}{c}-240 \zeta(3) \\
+360 \pi i \zeta(2)\end{array}$ & $-360 \zeta(2)$ & $-30 \pi i$ & \\
\hline 6 & $\begin{array}{c}6615 \zeta(6)-1120 \zeta(3)^{2} \\
+\pi i(3360 \zeta(2) \zeta(3)-2016 \zeta(5))\end{array}$ & $\begin{array}{c}2016 \zeta(5)-10080 \zeta(2) \zeta(3) \\
+3780 i \pi \zeta(4)\end{array}$ & $\begin{array}{c}-9450 \zeta(4) \\
-1680 i \pi \zeta(3)\end{array}$ & $\begin{array}{c}560 \zeta(3) \\
-840 \pi i \zeta(2)\end{array}$ & $630 \zeta(2)$ & $42 \pi i$ \\
\hline
\end{tabular}

Table 2. Numerically determined coefficients $\lambda_{k}^{(l)}$ for the equal mass Feynman integral w.r.t. the Frobenius basis $\varpi_{k}$ at the MUM point for $l \leq 6$.

Bessel expression is not valid and analytic continuation is needed. As seen from figure 3 we therefore have to analytically continue the solutions from $s=\frac{1}{(l+1)^{2}}$ to $s=0$. For this, the Bessel representation of $\mathcal{F}_{\sigma_{l}}$ is used to first fix the linear combination with respect to the local Frobenius basis ${ }^{19}$ around $s=\frac{1}{(l+1)^{2}}$. Then, by subsequent numerical analytic continuations along the upper half-plane, the local Frobenius basis around $s=\frac{1}{(l+1)^{2}}$ is related to the local Frobenius basis at the MUM point $s=0$. This numerically yields the desired coefficients $\lambda_{k}^{(l)}$ around $s=0$.

In order to guess the exact analytic expression of the coefficient $\lambda_{k}^{(l)}$ we take as an ansatz $^{20}$ all possible products of zeta values and $\pi$ that lead to a homogeneous transcendental weight of $l-k$ and linearly combine these products with rational coefficients to be determined. The latter are fitted by comparing the ansatz to the numerical values for $\lambda_{k}^{(l)}$ obtained in the previous paragraph. ${ }^{21}$ We checked these fits with 300 digits precision up to the loop order $l=17$ and with lower precision until $l=20$. For example, in the four-loop case we thus find

$$
\begin{array}{lll}
\lambda_{0}^{(4)}=-450 \zeta(4)-i \pi \cdot 80 \zeta(3) & \lambda_{1}^{(4)}=80 \zeta(3)-i \pi \cdot 120 \zeta(2) & \\
\lambda_{2}^{(4)}=180 \zeta(2) & \lambda_{3}^{(4)}=i \pi \cdot 20 & \lambda_{4}^{(4)}=-5 .
\end{array}
$$

Other examples for the first few loop orders $l \leq 6$, i.e., the coefficients $\lambda_{k}^{(l)}$, are displayed in table 2.

In all examples considered we observe that the imaginary part factors into $\pi$ and a sum of homogeneous transcendental weight $l-k-1$.

Moreover, all empirical results for $\lambda_{k}^{(l)}$ fit into the following combinatorial pattern: let $\mathcal{P}(l)$ be the set of integer partitions of $l$. From $\mathcal{P}(l)$ we take only the set of partitions $P(l)$

\footnotetext{
${ }^{19}$ These are obtained by shifting the variable $s$ to $\eta=s-\frac{1}{(l+1)^{2}}$ and solving the differential equation around $\eta=0$. It turns out that one obtains square root or logarithmic brunch cuts depending on whether $l$ is odd or even, respectively.

${ }^{20}$ This ansatz is inspired by the $\widehat{\Gamma}$-conjecture, which will be addressed in the next section.

${ }^{21}$ If we write down the ansatz with even powers of $\pi$ instead of even zeta values the coefficients in the ansatz turn out to be integers.
} 
with the property that any partition $p \in P(l)$ is given by a single even integer $g$, possibly zero, and $s$ odd integers ${ }^{22} o_{i}$ with $1<o_{1}<o_{2}<\cdots<o_{s}$ such that

$$
l=g+\sum_{i=1}^{s} m_{i} o_{i}
$$

so $m_{i}$ is the multiplicity of $o_{i}$ in the respective partition of $l$. In this case we may write $p=\left(g, o_{1}^{m_{i}}, \ldots, o_{s}^{m_{s}}\right)$. With this notation the combinatorial pattern of the coefficients $\lambda_{k}^{(l)}$, where $k=0, \ldots, l$, now reads ${ }^{23}$

$$
\begin{aligned}
\lambda_{k}^{(l)}= & (-1)^{k+1} \frac{(l+1) !}{k !} \sum_{p \in P(l-k)} \frac{(-1)^{\frac{g}{2}}(\pi)^{g}}{g !} \prod_{i=1}^{s} \frac{2^{m_{i}}}{\left(o_{i}\right)^{m_{i}} m_{i} !} \zeta\left(o_{i}\right)^{m_{i}} \\
& +i \pi(-1)^{k+1} \frac{(l+1) !}{k !} \sum_{p \in P(l-k-1)} \frac{(-1)^{\frac{g}{2}}(\pi)^{g}}{(g+1) !} \prod_{i=1}^{s} \frac{2^{m_{i}}}{\left(o_{i}\right)^{m_{i}} m_{i} !} \zeta\left(o_{i}\right)^{m_{i}}
\end{aligned}
$$

Indeed, we can give a generating function for the values $\lambda_{0}^{(l)}$ by

$$
\sum_{l=0}^{\infty} \lambda_{0}^{(l)} \frac{x^{l}}{(l+1) !}=-\mathrm{e}^{i \pi x+\sum_{k=1}^{\infty} \frac{2 \zeta(2 k+1)}{2 k+1} x^{2 k+1}}=-\frac{\Gamma(1-x)}{\Gamma(1+x)} \mathrm{e}^{-2 \gamma x+i \pi x},
$$

where $\gamma$ is the Euler-Mascheroni constant. The other coefficients are related to $\lambda_{0}^{(l)}$ by

$$
\lambda_{k}^{(l)}=(-1)^{k}\left(\begin{array}{c}
l+1 \\
k
\end{array}\right) \lambda_{0}^{(l-k)} .
$$

These findings conjecturally determine all $l$-loop banana Feynman integrals - in fact in all regions of their physical parameter space, since the linear combinations for the non-equal mass case follow by a simple symmetric splitting (see table 4 below).

Finally, we remark that $\sum_{k=0}^{l} \operatorname{Im}\left(\lambda_{k}^{(l)}\right) \varpi_{k}$ is proportional to the vanishing period at $s=\frac{1}{(l+1)^{2}}$, i.e., at the nearest conifold point, as seen from the MUM point. This numerical fact will get an interpretation and independent verification in section 4 , where homological mirror symmetry relates this period to a certain $K$-theory charge $Z_{D_{l-1}}$ associated with the mirror dual Calabi-Yau space (see eq. (4.19)).

\subsection{Monodromy}

To each singular point $s^{\prime}$ of $\mathcal{L}_{l}$ (recall eq. (3.7)) we can associate a monodromy matrix $M_{s^{\prime}}^{(l)}$ acting on the Frobenius basis $\varpi_{0}, \ldots, \varpi_{l}$ around the MUM point $s=0$ by

$$
\left(\begin{array}{c}
\varpi_{0} \\
\vdots \\
\varpi_{l}
\end{array}\right) \longmapsto M_{s^{\prime}}^{(l)}\left(\begin{array}{c}
\varpi_{0} \\
\vdots \\
\varpi_{l}
\end{array}\right),
$$

\footnotetext{
${ }^{22}$ Here $s$ stands for any appropriate non-negative integer, that is of course not to be confused with the momentum parameter $s=1 / t$.

${ }^{23}$ With $\frac{(\pi i)^{2 n}}{(2 n) !}=-\frac{\zeta(2 n)}{2^{2 n-1} B_{2 n}}$ we could also write the whole expression (3.18) in terms of zeta values and Bernoulli numbers.
} 
where we choose the analytic continuation along the upper half-plane and encircle the singular point $s^{\prime}$ counterclockwise. For the MUM point $s^{\prime}=0$ one can directly read off $M_{0}^{(l)}$ from the structure of the Frobenius basis. At the singular points

$$
\frac{1}{(l+1)^{2}}, \ldots, \frac{1}{\left(l+1-2\left\lfloor\frac{l+1}{2}\right\rfloor\right)^{2}}
$$

the local Frobenius basis can in each case be chosen so that only one solution is singular, i.e. for these points the monodromy satisifies

$$
\operatorname{dim}\left(\operatorname{image}\left(M_{s^{\prime}}^{(l)}-\mathbb{1}\right)\right)=1 .
$$

This motivates the definition of the Frobenius constants $\kappa_{k}^{\left(l, s^{\prime}\right)}$ by

$$
\left(M_{s^{\prime}}^{(l)}-\mathbb{1}\right) \varpi_{k}=\kappa_{k}^{\left(l, s^{\prime}\right)}\left(M_{s^{\prime}}^{(l)}-\mathbb{1}\right) \varpi_{0},
$$

i.e. we choose the normalization such that $\kappa_{0}^{\left(l, s^{\prime}\right)}=1$. Note that this only works when $\varpi_{0}$ is not invariant under $M_{s^{\prime}}^{(l)}$.

The Feynman integral $\mathcal{F}_{\sigma_{l}}$ is only singular at $s^{\prime}=0$ and $s^{\prime}=\frac{1}{(l+1)^{2}}$. For all other singular points $s^{\prime}$ satsifying (3.23) this implies ${ }^{24}$ that the Frobenius constants are constrained by

$$
\sum_{k=0}^{l} \lambda_{k}^{(l)} \kappa_{k}^{\left(l, s^{\prime}\right)}=0
$$

This can not hold for $s^{\prime}=\frac{1}{(l+1)^{2}}$. However, numerically we find even stronger conditions for this point, i.e. we observe that the Frobenius constants do not depend on the loop order,

$$
\kappa_{k}^{\left(l, 1 /(l+1)^{2}\right)}=\kappa_{k}^{\left(l^{\prime}, 1 /\left(l^{\prime}+1\right)^{2}\right)} \quad \text { for } k \leq l, l^{\prime}
$$

and that

$$
\sum_{k=0}^{l} \kappa_{k}^{\left(l, 1 /(l+1)^{2}\right)} \lambda_{k}^{(l)}=-(2 \pi i)^{l} .
$$

Thus, restricting to the singular point $s^{\prime}=\frac{1}{(l+1)^{2}}$, there is a series $\kappa_{k}$ of Frobenius constants determined by

$$
\sum_{k=0}^{\infty} \frac{\kappa_{k}}{k !} x^{k}=\frac{1}{\Gamma(1+x)^{2}} \mathrm{e}^{-2 \gamma x}
$$

In terms of these Frobenius constants we can write the associated monodromy matrix as

$$
M_{1 /(l+1)^{2}}^{(l)}=\mathbb{1}+\left(\begin{array}{c}
\kappa_{0} \\
\vdots \\
\kappa_{l}
\end{array}\right)\left(\delta_{0}^{(l)}, \ldots, \delta_{l}^{(l)}\right)
$$

\footnotetext{
${ }^{24}$ Insert the definitions $(3.15)$ and $(3.24)$ in the trivial monodromy condition $\left(M_{s^{\prime}}^{(l)}-\mathbb{1}\right) \mathcal{F}_{\sigma_{l}}=0$.
} 


\begin{tabular}{|lc|}
\hline Relation & Proof \\
\hline $\mathcal{F}_{\sigma_{1}}(1)=2 L\left(\left(\frac{\dot{3}}{3}\right), 1\right)=\frac{2 \pi}{3^{3 / 2}}$ & Bailey et al.: [41] \\
$\mathcal{F}_{\sigma_{2}}(1)=\left(1-2^{-2}\right) \zeta(2)=\frac{\pi^{2}}{4}$ & Bailey et al.: [41] \\
$\mathcal{F}_{\sigma_{3}}(1)=\frac{12 \pi}{\sqrt{15}} L\left(f_{3}, 2\right)=\frac{1}{30 \sqrt{5}} \prod_{k=0}^{3} \Gamma\left(\frac{2^{k}}{15}\right)$ & Zhou: [53] \\
$\mathcal{F}_{\sigma_{4}}(1)=8 \pi^{2} L\left(f_{4}, 2\right)$ & Zhou: [53] \\
$\mathcal{F}_{\sigma_{6}}(1)=144 \pi^{2} L\left(f_{6}, 4\right)$ & Zhou: [53] \\
\hline
\end{tabular}

Table 3. Special values of the equal mass Feynman integrals at $t=1$.

for some constants $\delta_{k}^{(l)}$. These constants can now be determined using the fact ${ }^{25}$

$$
M_{0}^{(l)} M_{1 /(l+1)^{2}}^{(l)} \mathcal{F}_{\sigma_{l}}=\mathcal{F}_{\sigma_{l}},
$$

which gives

$$
\sum_{l=1}^{\infty} \frac{\delta_{0}^{(l)}}{(l+1) !} x^{l}=-\frac{x}{\Gamma\left(1-\frac{x}{2 \pi i}\right)^{2}} \mathrm{e}^{\gamma \frac{x}{i \pi}}
$$

and the relation

$$
\delta_{k}^{(l)}=\frac{1}{(2 \pi i)^{k}}\left(\begin{array}{c}
l+1 \\
k
\end{array}\right) \delta_{0}^{(l-k)} .
$$

\subsection{Special points}

Special Feynman integrals can evaluate to interesting values related to critical L-values. Many relations of this type were conjectured by Broadhurst in [47] and some also have been proven later. To summarize some of these we define the Hecke eigenforms

$$
\begin{array}{ll}
f_{3}(\tau)=(\eta(3 \tau) \eta(5 \tau))^{3}+(\eta(\tau) \eta(15 \tau))^{3} & \in S_{3}\left(\Gamma_{0}(15),\left(\frac{-15}{\cdot}\right)\right)^{\text {new }} \\
f_{4}(\tau)=(\eta(\tau) \eta(2 \tau) \eta(3 \tau) \eta(6 \tau))^{2} & \in S_{4}\left(\Gamma_{0}(6)\right)^{\text {new }} \\
f_{6}(\tau)=\left(\frac{\eta(2 \tau)^{3} \eta(3 \tau)^{3}}{\eta(\tau) \eta(6 \tau)}\right)^{3}+\left(\frac{\eta(\tau)^{3} \eta(6 \tau)^{3}}{\eta(2 \tau) \eta(3 \tau)}\right)^{3} \in S_{6}\left(\Gamma_{0}(6)\right)^{\text {new }}
\end{array}
$$

in terms of the Dedekind eta function $\eta$. For the equal mass banana diagrams there are then the following proven relations for the on-shell point $s=1$ and loop orders $l \leq 6$ :

As was explained to us by Matt Kerr, ${ }^{26}$ since the banana Feynman integral is always a higher normal function (following from arguments similar to those in [54]) and its Milnor symbol becomes torsion at $t=1$ for any loop order $l$, the integral evaluates (up to factors of

\footnotetext{
${ }^{25}$ The combined path of analytic continuation corresponding to the left hand side of (3.30) is contractible in $\mathbb{P}^{1}$, as $\mathcal{F}_{\sigma_{l}}$ picks up no non-trivial monodromies at the other singular points.

${ }^{26}$ Private communication.
} 
$2 \pi i$ ) to a period of the underlying Calabi-Yau manifold. For modular Calabi-Yau manifolds it is expected that these periods evaluate to periods of the associated modular forms. For instance, $f_{3}$ is associated with a $\mathrm{K} 3$ surface of [55] and $f_{4}$ is associated with a rigid CalabiYau threefold [56].

Besides rigid Calabi-Yau three-folds defined over $\mathbb{Q}$, there are other interesting special Calabi-Yau threefolds $X$ associated with the four-loop diagram and suitable values of the kinematic parameters. One example are rank two attractor varieties. These are defined by demanding that their rational middle cohomology splits to

$$
H^{3}(X, \mathbb{Q})=\Lambda \oplus \Lambda_{\perp}
$$

with

$$
\Lambda \subset H^{(3,0)}(X) \oplus H^{(0,3)}(X) \quad \text { and } \quad \Lambda_{\perp} \subset H^{(2,1)}(X) \oplus H^{(1,2)}(X) .
$$

For the four-loop banana diagram, Candelas et al. found in [57] that the points $s=-1 / 7$ and $s=33 \pm 8 \sqrt{17}$ correspond to rank two attractor varieties. For all of these points the Hasse-Weil zeta function gives rise to modular forms of weight two and four and in [58] it was established numerically that the period matrices can be completely expressed in terms of periods and quasiperiods of these modular forms. E.g. for $s=-1 / 7$ one finds that the associated period matrix $T_{-1 / 7}$ can be written as

$$
T_{-1 / 7}=A\left(\begin{array}{cccc}
\omega_{4}^{+} & \eta_{4}^{+} & 0 & 0 \\
\omega_{4}^{-} & \eta_{4}^{-} & 0 & 0 \\
0 & 0 & \tilde{\omega}_{2}^{+} & \tilde{\eta}_{2}^{+} \\
0 & 0 & \tilde{\omega}_{2}^{-} & \tilde{\eta}_{2}^{-}
\end{array}\right) B
$$

where $A$ and $B$ are matrices with rational entries, $\omega_{4}^{ \pm}$and $\eta_{4}^{ \pm}$are periods and quasiperiods of a modular form of weight four and $\tilde{\omega}_{2}^{ \pm}$and $\tilde{\eta}_{2}^{ \pm}$are (up to a factor of $2 \pi i$ ) periods and quasiperiods of a modular form of weight two. The same is true for the other two points $s=33 \pm 8 \sqrt{17}$. However, in this case one has to take linear combinations over $\mathbb{Q}[\sqrt{-1}, \sqrt{-2}, \sqrt{17}]$ due to the coefficient fields of the modular forms and the irrationality of $s$, as explained in more detail in [58].

For the Feynman integral this means that the maximal cut integral at these points can be expressed in terms of the associated periods and quasiperiods. However, we found that this does not hold for the complete Feynman integral and it would be interesting to find out if and how this is related to the modularity of the underlying Calabi-Yau threefold.

\section{The $\widehat{\Gamma}$-class and zeta values at the point of maximal unipotent mon- odromy}

In subsection 2.1 we introduced a series of complete intersection Calabi-Yau geometries $W_{l-1}$ embedded in Fano varieties $F_{l}$ whose $l-1$ moduli correspond to the physical parameters $^{27}$ of the Feynman integral $\mathcal{F}_{\sigma_{l}}$, which is defined as a relative period on the mirror

\footnotetext{
${ }^{27}$ We drop from now on the superscript expressing the number of moduli.
} 
Calabi-Yau $M_{l-1}$. In this section we develop an application of the $\widehat{\Gamma}$-class formalism to these geometries. Technically, the resulting $\widehat{\Gamma}$-class formalism fixes the coefficients of the expansion of the Feynman integral (3.15) in terms of a canonical Frobenius basis of solutions of the GKZ system near the point of maximal unipotent monodromy given in subection 3.3. More precisely, the $\widehat{\Gamma}$-class allows to express the period integrals over a basis of geometric cycles with integral intersection form in the middle dimensional homology $H_{l-1}\left(M_{l-1}, \mathbb{Z}\right)$ and the Feynman integral, that corresponds to a unique geometric chain integral extension of this geometric basis, exactly in terms of linear combinations of this Frobenius basis. This in turn allows for the evaluation of the geometric integrals of interest to arbitrary precision in the high energy region. Conceptually, the $\widehat{\Gamma}$-class explains the inferred high energy expansion of Feynman integrals by relating the expansion coefficients (3.19), (3.20) and (5.17) to topological invariants of the proposed Calabi-Yau geometries and therefore confirms them to all loop orders.

Generally, the occurrence of powers of zeta values and of some special numbers in table 2 that can be identified with Euler number integrals over combinations of top Chern classes, as well as eq. (3.20), already hint that the coefficients come from a $\widehat{\Gamma}$-expansion integrated against the exponential $e^{t \omega}$ of a suitable Kähler form $\omega$. Below we use the relevant Calabi-Yau varieties $M_{l-1}$ and $W_{l-1}$ together with the Fano geometry $F_{l}$ to prove the equations (3.18), (3.19) and (5.17) using the $\widehat{\Gamma}$-class formalism.

For the benefit of the reader we give in the following paragraph some background on the definition of the $\widehat{\Gamma}$-classes. However, we want to emphasize that the definition of $K$-theory charges of branes on $W_{l-1} \subset F_{l}$ via the $\widehat{\Gamma}$-classes in (4.3) and (4.20) as well as the identification of these charges with the periods over an integral basis on $M_{l-1}$ and the chain integral using the mirror map (4.4), which follows from Givental, Lian and Yau's proof of mirror symmetry, is sufficient to understand the relation between the large energy asymptotics of the Feynman integral and the topological data of $W_{l-1}$ and hence the equations (3.18), (3.19) and (5.17).

The developments $[17,59-63]$ leading to the definition of the $\widehat{\Gamma}$-class followed a main theme in mathematics, namely to assign to objects from algebraic- or differential geometry simple topological invariants by which they can be distinguished or eventually even classified in the appropriate category. In our case this is the category of complex Kähler manifolds together with their category of coherent sheaves, which in the Calabi-Yau case are related by mirror symmetry to periods of their mirrors. The $\widehat{\Gamma}$-class is a refinement of the well known multiplicative characteristic classes that appear in the context of algebraic manifolds, namely the Chern characters, the $\hat{A}$-class and the Todd class defined from

$$
\operatorname{ch}(x)=e^{x}, \quad \hat{A}(x)=\frac{x / 2}{\sinh (x / 2)}, \quad \text { and } \quad \operatorname{td}(x)=e^{\frac{x}{2}} \hat{A}(x)
$$

by expansion in the Chern classes of the holomorphic bundle or sheaf $\mathcal{F}$ under consideration. How to pass from one of the above functions $f(x)$, which define the multiplicative characteristic class, using the (formal) eigenvalues $\delta_{i}$ of the sheaf $\mathcal{F}$ and symmetric functions of them to expressions involving the Chern classes is described in the excellent book [64]. The $\widehat{\Gamma}$-class is defined analogously using the Euler Gamma function $\Gamma$ as [63]

$$
\widehat{\Gamma}(x)=\Gamma\left(1-\frac{x}{2 \pi i}\right) .
$$


It has therefore also an expansion in Chern characters or Chern classes of the sheaf $\mathcal{F}$ as made explicit in (4.5) and (4.6) below. The key motivation for defining this new class [63] was that it calculates the $K$-theory charge for objects in the derived category of coherent sheaves with complex $k$-dimensional support in terms of classical topological data, as defined in (4.3) below. The physical realizations of these objects are known as $D_{k}$-branes (of complex dimension $k$ ) embedded in a projective manifold and the $K$-theory charges are identified with the physically well known central charges $Z_{D_{k}}$ of the BPS $D_{k}$-branes as reviewed for example in [65]. A key insight motivating the definition (4.2) is that it can be viewed as the suitable square root of the $\hat{A}$-genus in the sense that $\hat{A}(\mathcal{F})=\widehat{\Gamma}(\mathcal{F}) \widehat{\Gamma}^{*}(\mathcal{F})$. For Calabi-Yau manifolds homological mirror symmetry relates the $K$-theory charges of the objects in the derived category of coherent sheaves to the charges of the objects in the Fukaya category, the special Lagrangian branes, which are given by period integrals [65, 66]. This relation can be explicitly and precisely identified at the point of maximal unipotent monodromy. It yields the very remarkable fact that the high energy degeneration of the Feynman integral, which is defined by a relative period of the Calabi-Yau manifold $M_{l-1}$, is completely determined by the topological data of the mirror Calabi-Yau geometry $W_{l-1}$ inside the Fano variety $F_{l}$, as we will make explicit below.

We start with the imaginary part of the numbers in (3.18) or table 2. The analytic continuation as well as the monodromy worked out in section 3.5 reveals that this combination of periods corresponds to the one that vanishes at the nearest conifold $s=1 /\left(\sum_{i=1}^{l+1} \xi_{i}\right)^{2}$. Geometrically here a sphere $S^{l-1}$ vanishes. The latter is in the integral middle cohomology of $M_{l-1}$ and by the Seidel-Thomas twist [67] it corresponds to the $D_{l-1}$ brane that wraps the full $(l-1)$-dimensional mirror Calabi-Yau space $W_{l-1}$ in the derived category of coherent sheaves on $W_{l-1}[65]$.

The $\widehat{\Gamma}$-class formalism ${ }^{28}$ [60-63] allows to calculate the $K$-theory charge $Z_{D_{k}}$ of any D-brane $D_{k}$ via

$$
Z_{D_{k}}(\mathfrak{t})=\int_{W_{l-1}} e^{\omega \cdot \mathfrak{t}} \widehat{\Gamma}\left(T W_{l-1}\right) \operatorname{Ch}\left(D_{k}\right)+\mathcal{O}\left(e^{\mathfrak{t}}\right)
$$

Here $\omega$ is the Kähler form of $W_{l-1}$ and $\omega \cdot \mathfrak{t}=\sum_{i=1}^{h^{1,1}} \omega_{i} \mathfrak{t}^{i}$ refers to an expansion of the latter in terms of Kähler parameters $\mathfrak{t}^{i}$ w.r.t. a basis $\omega_{i}$ of the Kähler cone of $W_{l-1} \cdot \operatorname{Ch}\left(D_{k}\right)$ defines a cohomology class that specifies the $D_{k}$ brane. In particular, for the top dimensional $D_{l-1}$ brane $\operatorname{Ch}\left(D_{l-1}\right)=1$. The mirror map at the point of maximal unipotent monodromy ${ }^{29}$

$$
\mathfrak{t}^{k}=\frac{1}{2 \pi i} \frac{\varpi_{1}^{k}}{\varpi_{0}}=\frac{1}{2 \pi i} \log \left(z_{k}\right)+\tilde{\Sigma}_{k}(z)
$$

allows to relate the latter to the corresponding period in the Frobenius basis. More precisely, the central charge $Z_{D_{l-1}}$ is identified with the period in question and the $\mathrm{t}^{k}$-expansion can be identified with the logarithmic expansion in the Frobenius basis. In particular, to

\footnotetext{
${ }^{28}$ First explanations of the $\zeta(3) \chi /(2(2 \pi i))^{3}$ and the $\int_{W} c_{2} \wedge \omega_{k} / 24$ values in the periods of three-folds as coming from derivates of the gamma function were made in [17]. See also [59].

${ }^{29} \mathrm{By} \varpi_{1}^{k}, k=1, \ldots, h^{l-2,1}\left(M_{l-1}\right)$ we denote all single logarithmic periods. If $h^{l-2,1}\left(M_{l-1}\right)=1$ we omit the upper index.
} 
prove the occurence of the imaginary terms in the first column of table 2 , we only need to expand the $\widehat{\Gamma}$-class of the tangent sheaf $T W_{l-1}$ of $W_{l-1}$ and integrate termwise.

More generally, one may consider the regularised $\widehat{\Gamma}$-class of a sheaf $\mathcal{F}$. For a sheaf of rank $n$ the latter is defined as the symmetric expansion $\widehat{\Gamma}(\mathcal{F})=\prod_{i=1}^{n} e^{\gamma \delta_{i}} \Gamma\left(1+\delta_{i}\right)$ in terms of the eigenvalues $\delta_{i}$ of $\mathcal{F}$, which in turn is re-expressed in terms of its Chern classes $c_{k}=s_{k}\left(\delta_{1}, \ldots, \delta_{n}\right)$, where the $s_{k}$ denote elementary symmetric polynomials (defined after eq. (4.12)). Here $\gamma$ is the Euler-Mascheroni constant and the $e^{\gamma \delta_{i}}$ factors are introduced to cancel $e^{-\gamma c_{1}(\mathcal{F})}$ terms that would arise from the first derivative of just the $\Gamma\left(1+\delta_{i}\right)$ factors. For practical purposes it is faster to first write a closed formula in terms of the Chern characters $\operatorname{ch}_{k}(\mathcal{F})$ of $\mathcal{F}$. That yields the regularized $\widehat{\Gamma}(\mathcal{F})$-class as

$$
\widehat{\Gamma}(\mathcal{F})=\exp \left(\sum_{k \geq 2}(-1)^{k}(k-1) ! \zeta(k) \operatorname{ch}_{k}(\mathcal{F})\right) .
$$

The transition to the Chern classes $c_{k}$ can be made by Newton's formula

$$
\operatorname{ch}_{k}=(-1)^{(k+1)} k\left[\log \left(1+\sum_{i=1}^{\infty} c_{i} x^{i}\right)\right]_{k},
$$

where $[\cdot]_{k}$ means to take the $k$ th coefficient (in $x$ ) of the expansion of the expression in the [.]-bracket. We will shortly explain how to integrate products of Chern classes for the complete intersection varieties $F_{l}$ and $W_{l-1}$.

Relating the Frobenius basis of $M_{l-1}$ to the basis of Kähler classes on $W_{l-1}$. Let us now apply the mirror symmetry formalism developed in [17] to the geometry $W_{l-1}$ in (2.6), embedded in the Fano variety $F_{l}$, and its mirror $M_{l-1}$. Here a canonical subfamily of (2.6) with $l+1$ complex structure deformations is identified with the mirror manifold $M_{l-1}$ to $W_{l-1}$.

We first want to establish that the Picard-Fuchs equations of $M_{l-1}$ and their solutions in the canonical Frobenius basis are indeed the same as the one that we derived for the Feynman graph in the physical parametrization (2.11). According to [17] the period solutions of complete intersections in toric ambient spaces are specified by $\ell$-vectors ${ }^{30}$ $\ell^{(k)}=\left(\ell_{01}^{(k)}, \ldots, \ell_{0 h}^{(k)} ; \ell_{1}^{(k)}, \ldots, \ell_{c}^{(k)}\right)$ for $k=1, \ldots, h^{l-2,1}\left(M_{l-1}\right)$. Here $h$ is the number of complete intersection constraints, $c$ is the number of homogeneous coordinates of the ambient space and the $\ell_{i}^{(k)}$ for $i=1, \ldots, c$, are the degrees of the constraints. In the case of $M_{l-1}$ we have $h=2, c=2(l+1)$ and the $\ell^{(k)}$ read

$$
\begin{aligned}
\ell^{(1)} & =(-1,-1 ; 1,1,0,0, \cdots, 0,0,0,0) \\
\ell^{(2)} & =(-1,-1 ; 0,0,1,1, \cdots, 0,0,0,0) \\
& \vdots \\
\ell^{(l)} & =(-1,-1 ; 0,0,0,0, \cdots, 1,1,0,0) \\
\ell^{(l+1)} & =(-1,-1 ; 0,0,0,0, \cdots, 0,0,1,1) .
\end{aligned}
$$

\footnotetext{
${ }^{30}$ The terminology of $\ell$-vectors employed here is of course not be confused with vectors that have $l$ components or the like, which is why have chosen a different symbol $\ell$.
} 
From these $\ell$-vectors one obtains a generalized Gelfand-Kapranov-Zelevinskŭ differential system with holomorphic solution

$$
\omega_{0}(\underline{z} ; \underline{\epsilon})=\sum_{n_{1}, \ldots, n_{l+1} \geq 0} c(\underline{n}+\underline{\epsilon}) \underline{z}^{\underline{n}+\epsilon} .
$$

Here the underlined quantities are $(l+1)$-tuples and the series coefficients $c(\underline{n})$ are determined by the $l+1 \ell$-vectors via

$$
c(\underline{n})=\frac{\prod_{j=1}^{2}\left(-\sum_{k=1}^{l+1} l_{0 j}^{(k)} n_{k}\right) !}{\prod_{i=1}^{2 l+2}\left(\sum_{k=1}^{l+1} l_{i}^{(k)} n_{k}\right) !} .
$$

The $c(\underline{n}+\underline{\epsilon})$ are as usual defined by promoting all factorials $*$ ! in $(4.9)$ to $\Gamma(*+1)$ and deforming each integer $n_{k}$ to $n_{k}+\epsilon_{k}$. In particular, the unique holomorphic solution at the point of maximal unipotent monodromy is given by

$$
\varpi_{0}(\underline{z})=\left.\omega_{0}(\underline{z} ; \underline{\epsilon})\right|_{\underline{\epsilon}=\underline{0}}=\sum_{n_{1}, \ldots, n_{l+1} \geq 0}\left(\begin{array}{c}
|n| \\
n_{1}, \ldots, n_{l+1}
\end{array}\right) \prod_{k=1}^{2+1} z_{k}^{n_{k}} .
$$

Comparing with (2.13) we see that the coordinates ${ }^{31} z_{k}$ are related to the physical coordinates by

$$
z_{k}=s \xi_{k}^{2} \quad \text { for } \quad k=1, \ldots, l+1 .
$$

The point here is that the period of $M_{l-1}$ given in (4.10) is up to the simple parameter redefinition (4.11) equivalent to (2.13) after multiplying with the physical variable $s$.

The other periods for both systems can be obtained by the Frobenius method, as described in subsection 3.3 for the Feynman graph period and in [17] for the periods of $M_{l-1}$. The basic idea is to take certain combinations of derivatives w.r.t. the deformation parameters, $L_{c}^{(r)}=\left.\sum_{j_{1}, \ldots, j_{r}} c_{j_{1}, \ldots, j_{r}} \partial_{\epsilon_{j_{1}}} \ldots \partial_{\epsilon_{j_{r}}} \omega_{0}(\underline{z} ; \underline{\epsilon})\right|_{\underline{\epsilon}=\underline{0}}$. In particular, the solutions $\varpi_{k}=L_{\delta_{k, j}}^{(1)} / 2 \pi i$, where $k=1, \ldots, h_{\text {hor prim }}^{l-2,1}\left(M_{l-1}\right)$ with $h_{\text {hor prim }}^{l-2,1}\left(M_{l-1}\right)=l+1$, are the single logarithmic solutions, which together with $\varpi_{0}$ determine the mirror map (4.4). It is maybe worthwhile to stress the double role of the $\ell$-vectors in mirror symmetry [17]. On the one hand, as Mori vectors of $W_{l-1}$, they fix the basis of the Kähler cone $\omega_{i}$, $i=1, \ldots, h_{\text {vert prim }}^{1,1}\left(W_{l-1}\right)$ of $W_{l-1}$. On the other hand via (4.9) they determine in particular the $h_{\text {hor prim }}^{l-2,1}\left(M_{l-1}\right)$ logarithmic solutions at the MUM point of $M_{l-1}$. This is the reason that the mirror map (4.4) correctly relates these logarithmic solutions to the basis of Kähler classes used in (4.3). It also implies that the higher logarithmic solutions are fixed by the topological data of $W_{l-1}$. Indeed, their numbers are inferred by the differential ideal reported in figure 4 and match the primitive vertical Hodge numbers of $W_{l-1}$ reported in eq. (4.17) and thus, by mirror symmetry, also match the primitive horizontal middle dimensional Hodge numbers of $M_{l-1}$. This is further discussed in [68, 69] for four-folds.

These identifications also confirm that (2.6) is the right mirror $W_{l-1}$ to the Calabi-Yau $(l-1)$-fold family $M_{l-1}$, whose periods, together with the unique chain integral extension, in the parametrization (4.11) describe the Feyman integral exactly and non-redundantly in the physical parameters.

\footnotetext{
${ }^{31}$ These coordinates are also related to the redundant parameters multiplying the generic monomials of the Newton polytope associated to the complete intersection Calabi-Yau, see [17] for a definition.
} 
Evaluation of Chern classes. As explained before, this mirror pair implies that the evaluation of the $\widehat{\Gamma}$-class for $W_{l-1}$ must reproduce the imaginary parts of (3.18). The evaluation can be done in terms of integrals over Chern classes, to which we now turn. It follows by the adjunction formula that the Chern classes $c_{k}$ of $W_{l-1}$ are given by the degree $k$ part of

$$
c_{k}\left(W_{l-1}\right)=\left[\frac{\prod_{i=1}^{l+1}\left(1+H_{i}\right)^{2}}{\left(1+\sum_{i=1}^{l+1} H_{i}\right)^{2}}\right]_{\operatorname{deg}(H)=k} .
$$

More precisely, since the hyperplane classes in each $\mathbb{P}^{1}$ fulfill $H_{i}^{2}=0$ we can express $c_{k}$ in terms of elementary symmetric polynomials $s_{k}(\underline{H})=\sum_{i_{1}<\ldots<i_{k}} H_{i_{1}} \cdots H_{i_{k}}$ as

$$
c_{k}\left(W_{l-1}\right)=(-1)^{k} k ! \sum_{j=0}^{k} \frac{(-2)^{j}(k+1-j)}{j !} s_{k}(\underline{H})=: \mathcal{N}_{k}^{W_{l-1}} s_{k}(\underline{H}) .
$$

Similarly, considering the power one of the normal bundle in the denominator of (4.12) (instead of two) we can write for the Chern classes of the ambient space

$$
c_{k}\left(F_{l}\right)=(-1)^{k} k ! \sum_{j=0}^{k} \frac{(-2)^{j}}{j !} s_{k}(\underline{H})=: \mathcal{N}_{k}^{F_{l}} s_{k}(\underline{H}) .
$$

Moreover, we notice that the integral of a top degree product of Chern classes $c_{k_{n}}$ over $X=W_{l-1}$ or $X=F_{l}$ is given by

$$
\int_{X} \prod_{n} c_{k_{n}}=(l+1) ! \prod_{n} \frac{\mathcal{N}_{k_{n}}^{X}}{k_{n} !} .
$$

Comments on the primitive vertical cohomology. Before we proceed, let us interlude with comments on the primitive vertical homology of $W_{l-1}$ as well as on that of $F_{l}$. Let us denote the homogenous coordinates of the $k$ th $\mathbb{P}_{k}^{1}$ by $\left[x_{k}: y_{k}\right]$. Then in general the independent divisor classes of $W_{l-1}$, and similarly those of $F_{l}$, are the respective restrictions of the hyperplane classes ${ }^{32} D_{k}=\left\{a x_{k}+b y_{k}=0\right\}$ in $\left(\mathbb{P}_{1}\right)^{l+1}$ with topology $\left(\mathbb{P}_{1}\right)^{l}$. For $F_{l}$ and $W_{l-1}$ they have topology $F_{l-1}$ and $W_{l-2}$, respectively. We also have $D_{k}^{2}=0$, $k=1, \ldots, l+1$, and the top intersections are encoded in the coefficients of the rings

$$
\mathcal{R}^{W_{l-1}}=2 \sum_{i_{1}<\ldots<i_{l-1}} D_{i_{1}} \ldots D_{i_{l-1}} \quad \text { and } \quad \mathcal{R}^{F_{l}}=\sum_{i_{1}<\ldots<i_{l}} D_{i_{1}} \ldots D_{i_{l}} .
$$

The $D_{k}$ generate the primitive part of the vertical cohomology of $\left(\mathbb{P}^{1}\right)^{l+1}$, which is in this case already the full vertical cohomology with dimensions $h^{k, k}\left(\left(\mathbb{P}^{1}\right)^{l+1}\right)=\left(\begin{array}{c}l+1 \\ k\end{array}\right)$, while for $F_{l}$ and $W_{l-1}$ this primitive part has respective dimensions

$$
h_{\text {vert prim }}^{k, k}\left(F_{l}\right)=\left\{\begin{array}{l}
\left(\begin{array}{c}
l+1 \\
k
\end{array}\right) \text { if } k<\left\lceil\frac{l}{2}\right\rceil \\
\left(\begin{array}{l}
l+1 \\
l-k
\end{array}\right) \text { otherwise }
\end{array} \text { and } h_{\text {vert prim }}^{k, k}\left(W_{l-1}\right)=\left\{\begin{array}{l}
\left(\begin{array}{c}
l+1 \\
k
\end{array}\right) \quad \text { if } k<\left\lceil\frac{l}{2}\right\rceil-1 \\
\left(\begin{array}{c}
l+1 \\
l-1-k
\end{array}\right) \text { otherwise }
\end{array} .\right.\right.
$$

\footnotetext{
${ }^{32}$ To ease the notation we denote the divisor classes on $W_{l-1}$ and $F_{l}$ again by $D_{k}$.
} 
For high dimensions the primitive part is much smaller than the full vertical cohomology. The latter fact can be easily seen by calculating via the Hirzebruch-Riemann-Roch index theorem [64] the elliptic genera $\chi_{k}=\sum(-1)^{q} h^{q, k}(X)$ by evaluating instead of the $\widehat{\Gamma}$-class the Todd class against $\operatorname{Ch}\left(\wedge^{k} T X\right)$.

According to $[68,69]$ this primitive part of the vertical cohomology of $W_{l-1}$ should be mirror dual to the primitive horizontal middle cohomology of $M_{l-1}$, which corresponds to solutions of those Picard-Fuchs equations as discussed in subsection 5.2. Luckily, it is only those solutions we need to describe the banana integrals, while the actual (full) vertical and horizontal cohomology groups are much bigger. For example for the differently polarized K3 surfaces called $M_{2}$ and $W_{2}$ we have $h_{\text {vert prim }}^{1,1}\left(W_{2}\right)=h_{\text {hor prim }}^{1,1}\left(M_{2}\right)=4$ inside the twenty-dimensional group $H_{1,1}(\mathrm{~K} 3)$.

The $D_{k}$ are dual to the rational curves $C_{k}=\mathbb{P}_{k}^{1}$ which span the Mori cone. The latter pair by integration $\int_{C_{j}} \omega_{i}=\delta_{i j}$ with the Kähler forms $\omega_{k}$ of the $\mathbb{P}_{k}^{1}$, which span the Kähler cone.

Consistency check via instanton counts. We shall give another interlude from the topological string perspective. To see e.g. that $M_{3}$ is really the mirror to $W_{3}$ we can check the mirror symmetry predictions at the level of the instantons. Using equations (4.7) and (4.16) we apply ${ }^{33}$ the formalism of [17] for the prepotential of the case at hand and get

$$
\begin{aligned}
\mathcal{F}^{\text {prep }}= & 2 \sum_{i<j<k} \mathfrak{t}^{i} \mathfrak{t}^{j} \mathfrak{t}^{k}+\sum_{i=k}^{5} \frac{24}{24} \mathfrak{t}^{k}-80 \frac{\zeta(3)}{2(2 \pi i)^{3}}+24 \operatorname{Li}_{3}\left(q_{1}\right)+24 \operatorname{Li}_{3}\left(q_{1} q_{2}\right) \\
& +112 \operatorname{Li}_{3}\left(q_{1} q_{2} q_{3}\right)+1104 \operatorname{Li}_{3}\left(q_{1} q_{2} q_{3} q_{4}\right)+19200 \operatorname{Li}_{3}\left(q_{1} q_{2} q_{3} q_{4} q_{5}\right) \\
& +24 \operatorname{Li}_{3}\left(q_{1}^{2} q_{2} q_{3}\right)+1104 \operatorname{Li}_{3}\left(q_{1}^{2} q_{2} q_{3} q_{4}\right)+45408 \operatorname{Li}_{3}\left(q_{1}^{2} q_{3} q_{4} q_{5}\right) \\
& +24 \operatorname{Li}_{3}\left(q_{1}^{2} q_{2}^{2} q_{3}\right)+2800 \operatorname{Li}_{3}\left(q_{1}^{2} q_{2}^{2} q_{3} q_{4}\right)+212880 \operatorname{Li}_{3}\left(q_{1}^{2} q_{2}^{2} q_{3} q_{4} q_{5}\right) \\
& +80 \operatorname{Li}_{3}\left(q_{1}^{2} q_{2}^{2} q_{3}^{2}\right)+14496 \operatorname{Li}_{3}\left(q_{1}^{2} q_{2}^{2} q_{3}^{2} q_{4}\right)+1691856 \operatorname{Li}_{3}\left(q_{1}^{2} q_{2}^{2} q_{3}^{2} q_{4} q_{5}\right) \\
& +122352 \operatorname{Li}_{3}\left(q_{1}^{2} q_{2}^{2} q_{3}^{2} q_{4}\right)+\ldots .
\end{aligned}
$$

Here we have given the prepotential up to terms of the order $\mathcal{O}\left(q^{9}\right)$ and up to terms obtained by permutations of the $q_{k}$, which in turn are defined by $q_{k}=\exp \left(2 \pi i t^{k}\right)$ and keep track of the (multi-)degree of a rational instanton contribution. Also $\operatorname{Li}_{k}(x)=\sum_{n=1} \frac{x^{n}}{n^{k}}$ denotes the polylogarithm and $\operatorname{Li}_{3}(x)$ subtracts the multi-covering contributions to the $g=0$ curves. The integral coefficients in front of $\operatorname{Li}_{3}\left(\underline{q}^{\underline{d}}\right)$ are denoted by $n_{0}^{\underline{d}}$. If the curves are smooth we have $n_{0}^{\underline{d}}=(-1)^{\operatorname{dim}\left(\mathcal{M}_{\underline{d}}\right)} e\left(\mathcal{M}_{d}\right)$, i.e., the coefficient is up to sign the Euler number of the moduli space $\mathcal{M}_{\underline{d}}$ of the rational curves of degree $\underline{d}$. We see that these instanton numbers are indeed as expected for $W_{3}$. For example each single degree one curve gives a contribution as $24 \mathrm{Li}_{3}\left(q_{1}\right)$. Since the moduli space of such a curve is the K3 over which the $\mathbb{P}^{1}$ is fibered we get indeed $n_{0}^{(1,0,0,0,0)}=(-1)^{2} \chi(\mathrm{K} 3)=24$. The geometry $W_{l-1}$ has an intriguing nested fibration structure. For example the K3 called $W_{2}$ is in

\footnotetext{
${ }^{33}$ This can be done with the program Instanton distributed with [17].
} 
four ways fibred by the elliptic curve $W_{1}$ over $\mathbb{P}_{k}^{1}$ for $k=1, \ldots, 4$. While the Calabi-Yau three-fold $W_{3}$ for $l=4$ is in five ways fibered ${ }^{34}$ with a $\mathrm{K} 3$ fiber of topology $W_{2}$, etc.

Asymptotics of the banana integral. Now we can come to the main point and use the mirror picture, the $\widehat{\Gamma}$-class conjecture and the evaluation of the Chern classes to show that the leading logarithms (or $\mathfrak{t}$ powers) in the evaluation of the $\widehat{\Gamma}$-class

$$
Z_{D_{l-1}}(\mathfrak{t})=\int_{W_{l-1}} e^{\omega \cdot \mathfrak{t}} \widehat{\Gamma}\left(T W_{l-1}\right)+\mathcal{O}\left(e^{\mathfrak{t}}\right)
$$

yield precisely the imaginary parts of table 2, or more generally of (3.18).

Furthermore, we find that the leading logarithms (or $\mathfrak{t}$ powers) in the evaluation of

$$
Z_{\mathcal{F}_{\sigma_{l}}}(\mathfrak{t})=\int_{F_{l}} e^{\omega \cdot \mathfrak{t}} \frac{1}{\widehat{\Gamma}^{2}\left(T F_{l}\right)} \widehat{A}\left(T F_{l}\right)+\mathcal{O}\left(e^{\mathfrak{t}}\right)=\int_{F_{l}} e^{\omega \cdot \mathfrak{t}} \frac{\Gamma\left(1-c_{1}\right)}{\Gamma\left(1+c_{1}\right)} \cos \left(\pi c_{1}\right)+\mathcal{O}\left(e^{\mathfrak{t}}\right)
$$

yield precisely the real part of table 2, or more generally of (3.18). Here $\widehat{A}\left(T F_{l}\right)$ is the $\widehat{A}$-genus of the tangent sheaf $T F_{l}$, which is generally defined for a rank $n$ sheaf $\mathcal{F}$ as the symmetric expansion $\widehat{A}(\mathcal{F})=\prod_{i=1}^{n} \frac{2 \pi i \delta_{i}}{\sinh \left(2 \pi i \delta_{i}\right)}$ in terms of the eigenvalues $\delta_{i}$ of $\mathcal{F}$ and rewritten in terms of the Chern classes $c_{k}$. After the preprint appeared Hiroshi Iritani [71] confirmed that this application of the $\widehat{\Gamma}\left(T F_{l}\right)$ class to the asymptotics of the integral (2.1) is correct, as can be proven using the formalism in [61, 63].

Some remarks about (4.20) have to be said. First of all the two equalities in (4.20) were found by fitting an ansatz of a generalization of the $\widehat{\Gamma}$-class to the analytically computed values in (3.18). By this we observed that ansatze as in (4.20) reproduce the correct $\lambda$-coefficients. Notice that we are pretty sure that our ansatze are very special to the Fano variety $F_{l}$ we are considering here. We do not believe that the relations in (4.20) are generally true. We leave it to future work to geometrically interpret and prove our modified $\widehat{\Gamma}$-class conjecture, see also a related discussion in sections 9 and 10 of [72]. Secondly, we remark that the expected usual $\widehat{\Gamma}$-class ansatz as in (4.19) does not work even when ambiguities in the $\lambda$-coefficients are taken into account. These ambiguities arise due to analytic continuation of the Feynman integral around the MUM point. The monodromy of the Feynman integral is given by shifting each logarithm by $2 \pi i$ such that $\lambda$-coefficients which contain even zeta values are shifted, or in other words are ambiguous. Due to this ambiguity contributions containing only odd zeta values are ambiguity free and can naively be matched to the $\widehat{\Gamma}$-class. But also these terms are not correctly reproduced by a simple $\widehat{\Gamma}$-class. ${ }^{35}$ Therefore, we take into account the more general form as given in (4.20), which fits to all even and odd zeta values in the $\lambda$-coefficients. Moreover, notice that the second equality in (4.20) is very useful since from it the real part of (3.20) follows trivially because the integral over $F_{l}$ yields simply a contribution of $(l+1)$ ! for $c_{1}^{l}$. But this term is again canceled in the generating series (3.20).

\footnotetext{
${ }^{34}$ The latter fact can be checked using the criterium of Oguiso [70] from the topological data that appears in the classical part of $\mathcal{F}^{\text {prep }}$.

${ }^{35}$ For example for $l=9$ one may consider the contribution of $\zeta(3)^{3}$ or for $l=11$ the contribution of $\zeta(5) \zeta(3)^{2}$.
} 


\section{The l-loop non-equal mass banana Feynman integrals}

Having discussed the equal mass banana Feynman graph, we want to focus in this section on the full non-equal mass case. We give a general description and method how to compute the $l$-loop non-equal mass banana Feynman graph, exemplified by the four-loop non-equal mass case. In [16] we have already discussed the two- and three-loop case.

\subsection{Batyrev coordinates and the maximal cut integral}

As in [16] we use Batyrev coordinates ${ }^{36} z_{k}$, defined by

$$
z_{k}=\frac{\xi_{k}^{2}}{t-\sum_{i=1}^{l+1} \xi_{i}^{2}} \quad \text { for } k=1, \ldots, l+1 .
$$

Furthermore, we include in the Feynman integral $\mathcal{F}_{\sigma_{l}}\left(t, \xi_{i}\right)$ the additional factor

$$
a_{0}=t-\sum_{i=1}^{l+1} \xi_{i}^{2}=\frac{\xi_{l+1}^{2}}{z_{l+1}}
$$

which is related to the inner point of the polytope described by the polynomial constraint $P_{l}\left(t, \xi_{i} ; x\right)=0$. Then the expression we want to determine is

$$
\hat{\mathcal{F}}_{\sigma_{l}}=\int_{\sigma_{l}} \frac{a_{0} \mu_{l}}{P_{l}\left(t, \xi_{i} ; x\right)},
$$

and will $\hat{\mathcal{F}}_{T^{l}}$ be defined by analogy. For large momenta we can use the expression (2.13) to find the non-equal mass maximal cut Feynman integral ${ }^{37}$ including the inner point

$$
\hat{\varpi}_{0}\left(z_{i}\right)=\sum_{k_{1}, \ldots, k_{l+1} \geq 0}\left(\begin{array}{c}
|k| \\
k_{1}, \ldots, k_{l+1}
\end{array}\right)^{2}\left(\frac{1}{1+\sum_{i=1}^{l+1} z_{i}}\right)^{1+|k|} \prod_{i=1}^{l+1} z_{i}^{k_{i}} .
$$

Geometric series expansion gives a power series in the $z_{i}$ with non-negative exponents, valid if all $z_{k}$ are sufficiently small. The radius of convergence can be determined by the discriminant of the polynomial constraint $P_{l}(t, \xi ; x)$ or later also from the differential operators annihilating (5.4). We claim that the discriminant ${ }^{38}$ for the generic mass banana Feynman graph is given by

$$
\Delta\left(\mathcal{D}^{l}\right)\left(t, \xi_{i}\right)=t \prod_{\left\{T_{1}, T_{2}\right\} \in \mathcal{T}}\left[t-\left(\sum_{i \in T_{1}} \xi_{i}-\sum_{i \in T_{2}} \xi_{i}\right)^{2}\right],
$$

where the set

$$
\mathcal{T}=\left\{\left\{T_{1}, T_{2}\right\} \mid T_{1}, T_{2} \subseteq T^{(l+1)} \text { disjoint and } T_{1} \cup T_{2}=T^{(l+1)}\right\}
$$

\footnotetext{
${ }^{36}$ Notice that these are not the same Batyrev coordinates as defined in (4.11) which correspond to the complete intersection model. Here now we consider the hypersurface model with other Batyrev coordinates.

${ }^{37}$ Again up to normalization.

${ }^{38}$ Or at least the discriminant factors up to multiplicities.
} 
gives all possibilities to distribute the $l+1$ indices among two subsets (identifying swaps of the two sets). We have explicitly checked $\Delta\left(\mathcal{D}^{l}\right)\left(t, \xi_{i}\right)$ for $l=2,3,4$. The discriminant in the three-loop case is for instance given by

$$
\begin{aligned}
\Delta\left(\mathcal{D}^{3}\right)\left(t, \xi_{i}\right)= & t\left(t-\left(-\xi_{1}+\xi_{2}+\xi_{3}+\xi_{4}\right)^{2}\right)\left(t-\left(+\xi_{1}-\xi_{2}+\xi_{3}+\xi_{4}\right)^{2}\right) \\
& \cdot\left(t-\left(+\xi_{1}+\xi_{2}-\xi_{3}+\xi_{4}\right)^{2}\right)\left(t-\left(+\xi_{1}+\xi_{2}+\xi_{3}-\xi_{4}\right)^{2}\right) \\
& \cdot\left(t-\left(-\xi_{1}-\xi_{2}+\xi_{3}+\xi_{4}\right)^{2}\right)\left(t-\left(-\xi_{1}+\xi_{2}-\xi_{3}+\xi_{4}\right)^{2}\right) \\
& \cdot\left(t-\left(-\xi_{1}+\xi_{2}+\xi_{3}-\xi_{4}\right)^{2}\right)\left(t-\left(+\xi_{1}+\xi_{2}+\xi_{3}+\xi_{4}\right)^{2}\right) .
\end{aligned}
$$

In the equal mass case the discriminant $\Delta\left(\mathcal{D}^{l}\right)\left(t, \xi_{i}\right)$ reproduces the correct factors as stated in (3.7).

\subsection{Differential equations for the non-equal mass case}

Having found the holomorphic power series (5.4) describing the maximal cut Feynman integral for small values of $z_{k}$, as in [16] we now want to find a set of (in)homogeneous differential equations for it. With the help of (5.4) it can be checked that the second order operators $^{39}$

$$
\mathcal{D}_{k}=\theta_{k}^{2}-z_{k}\left(\sum_{i=1}^{l+1} \theta_{i}-2 \theta_{k}\right)\left(1+\sum_{i=1}^{l+1} \theta_{i}\right)-z_{k}\left(\sum_{i=1}^{l+1} z_{i}-z_{k}\right)\left(1+\sum_{i=1}^{l+1} \theta_{i}\right)\left(1+\sum_{i=1}^{l+1} \theta_{i}\right)
$$

for $k=1, \ldots, l+1$ annihilate $\hat{\varpi}_{0}\left(z_{i}\right)$. Applying these operators to the full Feynman integral $\hat{\mathcal{F}}_{\sigma_{l}}$ and performing a numerical integration we find that the operators $\mathcal{D}_{k}$ are indeed homogeneous operators annihilating the full Feynman integral $\hat{\mathcal{F}}_{\sigma_{l}}$, see also section 2.3 in $[16]$.

It turns out that these operators are enough to determine all solutions needed for the Feynman integral $\hat{\mathcal{F}}_{\sigma_{l}}$ — including those from integrals over closed cycles as well as the additional solution arising due to the chain integral — once the correct structure of solutions is imposed. Recall that the $z_{i}$ are local coordinates around a MUM-point $\left(z_{i}=0\right.$ for all $i=1, \ldots, l+1)$, so there is a unique holomorphic solution up to normalization and the rest of the local Frobenius basis is spanned by solutions with increasing degree of the leading logarithms. ${ }^{40}$ For periods coming from closed cycles the highest degree in logarithms of the $l+1$ variables $z_{i}$ is given by $l-1$, the (complex) dimension of the Calabi-Yau variety. Since in an algebraically realized Calabi-Yau variety, which fixes a polarization, only the primitive part of the horizontal subspace of the middle cohomology

\footnotetext{
${ }^{39}$ At this point we want to mention that it is also possible the obtain differential operators from the complete intersection model in 2.1. They follow directly from the $\ell$-vector description, see [17], and are closely related to the ones in (5.8). The following discussion could have similarly been made also starting from these operators and analyzing the complete intersection model. Nevertheless, we focus in our discussion on the hypersurface model since this is how we originally invented our method.

${ }^{40}$ Here the notion of degree or better multidegree is such that for instance the periods $\hat{\varpi}_{k}^{r}$ in eqs. (5.11) have degree $r$, i.e. the arguments of the logs are irrelevant for this notion. Alternatively we call those $r$-fold logarithmic.
} 


$$
\begin{aligned}
& \text { \#hol } \# \log ^{1}{ }^{1} \# \log ^{1}
\end{aligned}
$$

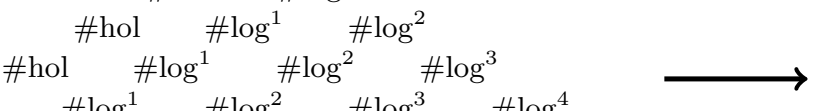

$$
\begin{aligned}
& \text { \#hol \# } \# \log ^{1} \# \log ^{2} \quad \# \log ^{3} \quad \# \log ^{4} \\
& \text { \#hol \# } \# \log ^{1} \quad \# \log ^{2} \quad \# \log ^{3} \quad \# \log ^{4} \quad \# \log ^{5}
\end{aligned}
$$

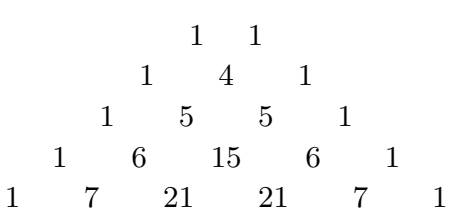

Figure 4. The number of logarithmic solution for the non equal mass case for $l=2, \ldots, 6$.

can be described by period integrals satisfying Picard-Fuchs equations [18], the number of $r$-fold logarithmic solutions corresponds to the dimension $h_{\text {hor prim }}^{(l-1-r)}$ of the respective piece in that subspace. Since the Feynman integral, i.e. the parametrization of the underlying Calabi-Yau variety, is completely symmetric in the $z_{i}$ variables, solutions always come in complete orbits under permutations of the $z_{i}{ }^{41}$ On these grounds it is already possible to propose a generalization of the $l=2,3,4$ results that we explicitly calculated. The number of periods over closed cycles is given by figure 4 . Here the entries in the $n$th row correspond to the numbers $d_{r}^{(l)}$ of basis elements at loop order $l=n+1$ that have the indicated degree $r$ in logarithms, coinciding with the length of an orbit under permutations of the $z_{i}$. The left side is Pascal's triangle and the right side is fixed by the invariance of the primitive part of the horizontal Hodge numbers ${ }^{42}$ of our variety under complex conjugation.

We shall further illucidate the combinatorial pattern that allows for a complete determation of a Frobenius basis and gives rise to the above numbers. After suitable normalization the holomorphic power series starts with unity

$$
\hat{\varpi}_{0}\left(z_{i}\right)=1+\mathcal{O}\left(z_{i}^{2}\right)
$$

For $l>2$ there are $l+1$ single logarithmic solutions of the form

$$
\hat{\varpi}_{1}^{k}\left(z_{i}\right)=\log \left(z_{k}\right)+\mathcal{O}\left(z_{i}\right)
$$

Solutions of higher logarithmic degree $r \leq\left\lceil\frac{l}{2}\right\rceil-1$ are of the form

$$
\hat{\varpi}_{r}^{k}\left(z_{i}\right)=\prod_{i=1}^{r} \log \left(z_{j_{i}(k)}\right)+\mathcal{O}\left(z_{i}\right) \quad \text { for some } \quad\left\{j_{1}^{(k)}, \ldots, j_{r}^{(k)}\right\} \in T_{r}^{(l+1)}
$$

where $k=1, \ldots,\left(\begin{array}{c}l+1 \\ r\end{array}\right)$ now labels the elements of $T_{r}^{(l+1)}$, which we recall is the set of all subsets of $\{1, \ldots, l+1\}$ of length $r$.

Further logarithmic solutions for $r>\left\lceil\frac{l}{2}\right\rceil-1$ are obtained as follows. Label the $\left(\begin{array}{c}l+1 \\ l-r-1\end{array}\right)$ subsets of $\{1, \ldots, l+1\}$ having exactly $l-r-1$ elements by $k$. For each $k$, i.e. a choice

\footnotetext{
${ }^{41}$ Similarly, the differential equations satisfied by the Feynman integrals always come in complete orbits.

${ }^{42}$ At that point we want to mention that Fernando Rodriguez Villegas told us that he actually found a motive from which one can directly calculate the horizontal Hodge numbers agreeing with our primitive horizontal Hodge numbers (4.17). We thank him for telling us this fact. Furthermore, we think that with some effort it is also possible to compute the full horizontal Hodge numbers from the complete intersection model described in section 2.1 .
} 
$N^{(k)}=\left\{n_{1}^{(k)}, \ldots, n_{l-r-1}^{(k)}\right\} \subseteq\{1, \ldots, l+1\}$, the solution $\hat{\varpi}_{r}^{k}$ only involves $r$-fold logarithms in the remaining $r+2$ variables and we have

$$
\hat{\varpi}_{r}^{k}\left(z_{i}\right)=\sum_{\left\{j_{1}, \ldots, j_{r}\right\} \in\{1, \ldots, l+1\} \backslash N^{(k)}} \prod_{i=1}^{r} \log \left(z_{j_{i}}\right)+\mathcal{O}\left(z_{i}\right) .
$$

As a consequence of these formulae, the total number of solutions (that correspond to integrals over closed cycles) is given by

$$
\sum_{r=0}^{l-1} d_{r}^{(l)}=2^{l+1}-\left(\begin{array}{c}
l+2 \\
\left\lfloor\frac{l+2}{2}\right\rfloor
\end{array}\right) .
$$

The additional (special) solution can be chosen to start as

$$
\hat{\varpi}_{l}\left(z_{i}\right)=\prod_{i=1}^{l+1} \log \left(z_{i}\right) \sum_{i=1}^{l+1} \frac{1}{\log \left(z_{i}\right)}+\mathcal{O}\left(z_{i}\right) .
$$

A generating function. As in the equal mass case we can define a generating function for a set of solutions by shifting $k_{i} \rightarrow k_{i}+\epsilon_{i}$ in the series (5.4). Derivatives with respect to the formal parameters $\epsilon_{i}$ then yield the higher logarithmic solutions. One has to take care that if the degree of the logarithms is larger than one, appropriate linear combinations of various derivatives have to be taken to get a correct solution. That is, these linear combinations should have the same combinatorial structure as the logarithmic solutions in (5.11), (5.12) and (5.14).

Loop reduction. An interesting feature of the solutions presented here is the following reduction property. Starting from those solutions for the $l$-loop banana integral which have no contribution from $\log \left(z_{l+1}\right)$, those solutions of the $(l-1)$-loop integral which correspond to closed cycles are obtained from the former by setting $z_{l+1}$ to zero. In this limit some $l$-loop solutions vanish and the number of non-zero logarithmic solutions of the $(l-1)$-loop geometry thus obtained nicely matches the number one expects according to the structure (5.9)-(5.12).

A four-loop example. To illustrate the structure of the solutions we consider the fourloop case, which is the lowest loop order that, to our knowledge, has not been treated analytically in the non-equal mass case in the literature. Solutions start with

$$
\begin{aligned}
\hat{\varpi}_{0}\left(z_{i}\right)= & 1+2\left(z_{1} z_{2}+z_{1} z_{3}+z_{2} z_{3}+z_{1} z_{4}+z_{2} z_{4}+z_{3} z_{4}+z_{1} z_{5}+z_{2} z_{5}\right. \\
& \left.+z_{3} z_{5}+z_{4} z_{5}\right)+\mathcal{O}\left(z_{i}\right)^{3} \\
\hat{\varpi}_{1}^{1}\left(z_{i}\right)= & \log \left(z_{1}\right)-z_{1}+z_{2}+z_{3}+z_{4}+z_{5}+\mathcal{O}\left(z_{i}\right)^{2} \\
\hat{\varpi}_{2}^{1}\left(z_{i}\right)= & \log \left(z_{2}\right) \log \left(z_{3}\right)+\log \left(z_{2}\right) \log \left(z_{4}\right)+\log \left(z_{3}\right) \log \left(z_{4}\right)+\log \left(z_{2}\right) \log \left(z_{5}\right) \\
& +\log \left(z_{3}\right) \log \left(z_{5}\right)+\log \left(z_{4}\right) \log \left(z_{5}\right)+\mathcal{O}\left(z_{i}\right)
\end{aligned}
$$




$$
\begin{aligned}
\hat{\varpi}_{3}\left(z_{i}\right)= & \log \left(z_{1}\right) \log \left(z_{2}\right) \log \left(z_{3}\right)+\log \left(z_{1}\right) \log \left(z_{2}\right) \log \left(z_{4}\right)+\log \left(z_{1}\right) \log \left(z_{3}\right) \log \left(z_{4}\right) \\
& +\log \left(z_{2}\right) \log \left(z_{3}\right) \log \left(z_{4}\right)+\log \left(z_{1}\right) \log \left(z_{2}\right) \log \left(z_{5}\right)+\log \left(z_{1}\right) \log \left(z_{3}\right) \log \left(z_{5}\right) \\
& +\log \left(z_{2}\right) \log \left(z_{3}\right) \log \left(z_{5}\right)+\log \left(z_{1}\right) \log \left(z_{4}\right) \log \left(z_{5}\right)+\log \left(z_{2}\right) \log \left(z_{4}\right) \log \left(z_{5}\right) \\
& +\log \left(z_{3}\right) \log \left(z_{4}\right) \log \left(z_{5}\right)+\mathcal{O}\left(z_{i}\right) \\
\hat{\varpi}_{4}\left(z_{i}\right)= & \log \left(z_{1}\right) \log \left(z_{2}\right) \log \left(z_{3}\right) \log \left(z_{4}\right)+\log \left(z_{1}\right) \log \left(z_{2}\right) \log \left(z_{3}\right) \log \left(z_{5}\right)+\mathcal{O}\left(z_{i}\right) \\
& +\log \left(z_{1}\right) \log \left(z_{2}\right) \log \left(z_{4}\right) \log \left(z_{5}\right)+\log \left(z_{1}\right) \log \left(z_{3}\right) \log \left(z_{4}\right) \log \left(z_{5}\right) \\
& +\log \left(z_{2}\right) \log \left(z_{3}\right) \log \left(z_{4}\right) \log \left(z_{5}\right)+\mathcal{O}\left(z_{i}\right),
\end{aligned}
$$

where the other single- and double-logarithmic solutions are obtained by replacing $z_{1} \leftrightarrow z_{i}$ by $i=2, \ldots, 5$.

Analytic continuation by completing the differential ideal. To extend the solutions $\hat{\varpi}_{n}\left(z_{i}\right)$ for $n=0, \ldots, l$ to other domains of the $z_{i}$-parameter space analytic continuation is needed. To this end it is necessary to have a complete set of differential equations. By these we mean a set of differential equations such that the number of corresponding solutions is equal to the number of solutions given in (5.13) plus the additional special solution. Notice that in general only the total number of solutions stays the same upon analytic continuation to other points. The precise logarithmic structure of the solutions changes, in particular for analytic continuation to non-singular points of the differential equations. With this (or these) additional differential equation(s) one can transform the local solutions of the MUM point to domains beyond the original domain of convergence by matching local Frobenius bases on overlapping regions. ${ }^{43}$

As we have explicitly seen in the two- and three-loop case ${ }^{44}$ some differential equations get even extended with inhomogeneities if the whole Feynman integral $\hat{\mathcal{F}}_{\sigma_{l}}$ should satisfy them instead of just the maximal cut $\hat{\mathcal{F}}_{T^{l}}$. For the four-loop case we have found a second order operator with coefficients being polynomials of multidegree three in the five variables $z_{i}$, leading to an inhomogeneous differential equation given in appendix B. It is hard to give a general formula for the additional and perhaps inhomogeneous differential equation(s). In fact, it is not even clear whether one has to extend the operators $\mathcal{D}_{k}$ just by a single differential equation with or without an inhomogeneity. The general strategy to get a complete system of (inhomogeneous) differential equations is simply that one searches for new ones until only the expected number of solutions is determined by these operators. We suggest to search for second order operators by systematically increasing the multidegree of the coefficient polynomials multiplying the derivatives. At some point these are expected to yield a complete set of differential equations, as we checked for $l \leq 4$. If not, one has to go to higher degree equations in the ansatz.

\footnotetext{
${ }^{43}$ Of course, if one had again sufficient information about the analytic structure of a local Frobenius basis in the new region, one could construct a basis despite only knowing an incomplete set of differential equations, as was the case for the MUM point.

${ }^{44}$ For the two- and three-loop case these (inhomogeneous) differential equations are listed in [16] and in the auxiliary Mathematica-file accompanying this paper, see http://www.th.physik. uni-bonn.de/Groups/ Klemm/data.php.
} 


\begin{tabular}{|c|c|c|c|c|c|}
\hline$l$ & $\lambda_{0, s}{ }^{(l)}$ & $\lambda_{1, s}{ }^{(l)}$ & $\lambda_{2, s}{ }^{(l)}$ & $\lambda_{3, s}{ }^{(l)}$ & $\lambda_{4, s}{ }^{(l)}$ \\
\hline 2 & $18 \zeta(2)$ & $2 \pi i$ & 1 & \multirow{5}{*}{1} & \\
\hline \multirow{4}{*}{3} & \multirow{4}{*}{$-16 \zeta(3)+24 \pi \zeta(2) i$} & $-18 \zeta(2)$ & & & \\
\hline & & $-18 \zeta(2)$ & $-2 \pi i$ & & \\
\hline & & $-18 \zeta(2)$ & & & \\
\hline & & $-18 \zeta(2)$ & & & \\
\hline \multirow{5}{*}{4} & \multirow{5}{*}{$-450 \zeta(4)-80 \pi \zeta(3) i$} & $16 \zeta(3)-24 \pi \zeta(2) i$ & $6 \zeta(2)$ & \multirow{5}{*}{$2 \pi i$} & \multirow{5}{*}{1} \\
\hline & & $16 \zeta(3)-24 \pi \zeta(2) i$ & $6 \zeta(2)$ & & \\
\hline & & $16 \zeta(3)-24 \pi \zeta(2) i$ & $6 \zeta(2)$ & & \\
\hline & & $16 \zeta(3)-24 \pi \zeta(2) i$ & $6 \zeta(2)$ & & \\
\hline & & $16 \zeta(3)-24 \pi \zeta(2) i$ & $6 \zeta(2)$ & & \\
\hline
\end{tabular}

Table 4. Coefficients giving the non-equal mass Feynman integral in the MUM point Frobenius basis of subsection 5.2 .

\subsection{Linear combination for the non-equal mass Feynman integral}

Next we fix the linear combination of the previously constructed solutions that gives the full non-equal mass Feynman integral

$$
\hat{\mathcal{F}}_{\sigma_{l}}=\sum_{r=0}^{l} \sum_{s=1}^{d_{r}^{(l)}} \lambda_{r, s}^{(l)} \hat{\varpi}_{r}^{s} .
$$

As in the equal mass case we numerically compute $\hat{\mathcal{F}}_{\sigma_{l}}$ to fix the coefficients $\lambda_{r, s}^{(l)}$. These again turn out to be appropriate combinations of zeta values, closely related to the equal mass ones $\lambda_{r}^{(l)}$. For $l=2,3,4$ and with respect to the basis of solutions given by (5.9), (5.10), (5.11), (5.12) and (5.14) we find the explicit values shown in table 4.

We see that with our choice of basis the equal mass values for $\lambda_{r}^{(l)}$ split symmetrically into the values $\lambda_{r, s}^{(l)}$. In general we claim that the non-equal mass values satisy

$$
\lambda_{r, s}^{(l)}= \begin{cases}\lambda_{r}^{(l)} \cdot\left(\begin{array}{c}
l+1 \\
r
\end{array}\right)^{-1} & \text { for } r \leq\left\lceil\frac{l}{2}\right\rceil-1 \text { and } s=1, \ldots,\left(\begin{array}{c}
l+1 \\
r
\end{array}\right) \\
\lambda_{r}^{(l)} \cdot\left(\left(\begin{array}{c}
l+1 \\
l-r-1
\end{array}\right)\left(\begin{array}{c}
r+2 \\
r
\end{array}\right)\right)^{-1} & \text { for } r>\left\lceil\frac{l}{2}\right\rceil-1 \text { and } s=1, \ldots,\left(\begin{array}{c}
l+1 \\
l-r-1
\end{array}\right) \\
1 & \text { for } r=l \quad \text { and } s=1 .\end{cases}
$$

We remark that the factor $\left(\begin{array}{c}r+2 \\ r\end{array}\right)$ results from the $\left(\begin{array}{c}r+2 \\ r\end{array}\right)$ terms in the sum of (5.12).

\section{Conclusion and outlook}

In this paper we used techniques developed in the interface of algebraic geometry and algebraic number theory with mathematical physics mostly in the context of string theory to describe the complete analytic structure of the $l$-loop banana integrals. While the low energy region was relatively well under control using the Bessel function integrals most of 
the results near the region $t \geq\left(\sum_{i=1}^{l+1} \xi_{i}\right)^{2}$ and into the high energy regime are new and related in a simple and beautiful way to the realization of the Feynman integrals as periods of very symmetric complete intersection Calabi-Yau $(l-1)$-folds and their extension.

In finding the most suitable geometry it turned out that the motivic perspective is as important as the geometrical intuition. So one lesson to keep in mind is to try first to find the motive based on any available exact period that one can get hands on, in the simplest possible or most suitable geometric realizations for the question at hand. For this task data bases for motives of Calabi-Yau- and Fano motives are very useful.

Due to the $\widehat{\Gamma}$-class evaluation the occurrence of products of zeta values in the large energy limit, whose highest degree of transcendentality is $l$, is by now explained for the banana graphs. However, while the numerical evidence is overwhelming our application of the $\widehat{\Gamma}$-class to the Fano variety (somewhat different as to the Calabi-Yau to get the imaginary part) is still conjectural and poses an interesting challenge to prove it in mathematical rigor. Nevertheless, the convincing evidence might shed light on the general observation that Feynman integrals evaluate at special points to interesting (conjecturally transcendental) numbers, such as multiple zeta values (MZV) or critical values of modular L-functions. For the latter fact we have found an interesting new example at the attractor points of the maximal cut integral. At this point the Galois representation splits relative to the generic Calabi-Yau family into two simple factors.

It might be interesting to relate this to more systematic studies of the degree of transcendentality and the motivic Galois group. For example for massless $\phi^{4}$-theory, a specific kind of Feynman periods is studied in [73], originating from primitive vacuum graphs more precisely, primitive logarithmically divergent graphs (with external legs) may be regularised, and the residue in the regulator is in [73] called the period of the graph (with amputated legs). This sort of period gives a renormalization scheme independent contribution to the $\beta$-function [74]. These massless $\phi^{4}$-periods have also served as a data mine for exploring the number theory content of perturbative QFT. Notably, the family of zig-zag graphs gives the only family of periods known to all loop orders, proven to be rational multiples of odd zeta values [75]. However, the number content of QFTs exceeds the span of multiple zeta values [76, 77]. There is a conjectured Galois coaction on the periods [78, 79], compatible with known $\phi^{4}$-periods and also with the number content [80] of the electron anomalous magnetic moment in QED.

The most practical consequence of the present work is that the GKZ methods and the understanding of the analytic structure of the integrals allow to calculate it extremely fast and to very high numerical precision or as simple series expansion. Furthermore, it is reasonable to have similar expectations for the period integrals of higher orders in the dimensional regularization parameter $\varepsilon .{ }^{45}$ It would be interesting to collaborate with complementary expertise to provide actual program tools for this task.

The very richly nested but, in each occasion, simple fibration structure going back all the way to the elliptic fibrations of the K3 surfaces in the geometry describing the Feynman

\footnotetext{
${ }^{45}$ We are tempted to speculate on the existence of differential operators in the moduli relating the $\varepsilon^{0}$-order integals (i.e. $d=2$ ) computed in this work and the higher terms in $\varepsilon$, similar to the calculations in [81, 82].
} 
integral, as mentioned in section 4, guarantees that there are all kinds of overlapping limits in which simpler functions, as for example the elliptic dilogarithm, are bound to occur $[11,83]$. In the context of large base parameter of K3 fibered Calabi-Yau threefolds it is known by heterotic Type II duality that exact modular functions obtained by the Borcherds lift do describe the integral instanton expansion completely [84-87]. This suggests that there will be a further confluence of techniques to understand more generally the automorphic and integral structures of the Feynman integrals.

\section{Acknowledgments}

It is a pleasure to thank Don Zagier for finding the generating functions for $\lambda$-values, commenting on the double Borel sum of the symmetric product of Bessel functions and collaboration on work related to periods and quasiperiods of modular forms. We further want to thank the members of the International Group de Travail on differential equations (in Paris), initiated by Vasily Golyshev to create a lively research environment during the pandemic, for most important remarks. In particular, Matt Kerr for crucial discussions about the geometry of the three-loop graph, to Fernando Rodriguez Villegas for providing an alternative motive for the periods over the primitive cohomology and Danylo Radchenko for sending us Sage code to calculate the corresponding Ehrhart-polynomials. To Claude Sabbah, Matt Kerr and Spencer Bloch for pointing out state of the art knowledge about the special values at $t=1$ using L-functions and Kloosterman sums. Special thanks to Claude Duhr for providing the physical motivations and a most valuable first hand perspective from the practitioner. We also would like to thank Hiroshi Iritani for a private communication confirming the validity of formula (4.20). Moreover, we want to thank Victor Batyrev, Yang-Hui He, Philip Candelas, Xenia de la Ossa, Mohamed Elmi, Amir Kashani-Poor, Duco van Straten and Hans Jockers for further help and discussions. Kilian Bönisch thanks the German Academic Scholarship Foundation for financial support. We also thank the Bonn-Cologne Graduate School of Physics and Astronomy for financial support.

\section{A Derivation of the Bessel function representation}

In this appendix we rewrite the Feynman integral (2.1) in terms of an integral over Bessel functions, closely follow the discussion in [22], see also [88].

Starting from (2.1) one expands a factor in the denominator in terms of a geometric series,

$$
\mathcal{F}_{\sigma_{l}}=-\sum_{k=0}^{\infty} t^{k} \int_{\sigma_{l}}\left(\frac{1}{\left(\sum_{i} \xi_{i}^{2} x_{i}\right)\left(\sum_{i} x_{i}^{-1}\right)}\right)^{k+1} \frac{\mu_{l}}{\prod_{i} x_{i}},
$$

converging if $t<\left(\sum_{i} \xi_{i}^{2} x_{i}\right)\left(\sum_{i} \frac{1}{x_{i}}\right)$. Since $x_{i} \geq 0$ we require

$$
t<\left(\sum_{i=1}^{l+1} \xi_{i}\right)^{2}
$$


for eq. (A.1) to hold. Furthermore, we can use the identity

$$
\left(\frac{1}{a}\right)^{k+1}=\frac{1}{k !} \int_{0}^{\infty} \mathrm{e}^{-a x} x^{k} \mathrm{~d} x,
$$

which is valid for $\operatorname{Re}(a)>0$ and $k>-1$, to rewrite the denominator in (A.1) introducing two new integrations

$$
\mathcal{F}_{\sigma_{l}}=-\sum_{k=0}^{\infty} \frac{t^{k}}{(k !)^{2}} \int_{\sigma_{l}} \int_{0}^{\infty} \int_{0}^{\infty} \mathrm{e}^{-u\left(\sum_{i} \xi_{i}^{2} x_{i}\right)-v\left(\sum_{i} \frac{1}{x_{i}}\right)} \frac{\mathrm{d} u \mathrm{~d} v}{u^{-k} v^{-k}} \frac{\mu_{l}}{\prod_{i} x_{i}} .
$$

The projective integral over $\sigma_{l}$ can be performed using the identity

$$
\int_{0}^{\infty} \mathrm{e}^{-u m^{2} x-\frac{v}{x}} \frac{\mathrm{d} x}{x}=2 K_{0}(2 m \sqrt{u v})
$$

involving the modified Bessel function of the second kind $K_{0}$. We obtain

$$
\mathcal{F}_{\sigma_{l}}=-2^{l} \sum_{k=0}^{\infty} \frac{t^{k}}{(k !)^{2}} \int_{0}^{\infty} \int_{0}^{\infty} \prod_{i=1}^{l} K_{0}\left(2 \xi_{i} \sqrt{u v}\right) \mathrm{e}^{-u \xi_{l+1}^{2}-v} \frac{\mathrm{d} u \mathrm{~d} v}{u^{-k} v^{-k}} .
$$

Introducing new variables $y=v$ and $z=2 \sqrt{u v}$ with $\mathrm{d} u \mathrm{~d} v=\frac{z}{2 y} \mathrm{~d} y \mathrm{~d} z$ and integrating subsequently over $y$ we find

$$
\mathcal{F}_{\sigma_{l}}=-2^{l+1} \sum_{k=0}^{\infty} \frac{t^{k}}{(k !)^{2}} \int_{0}^{\infty}\left(\frac{z}{2}\right)^{2 k+1} \prod_{i=1}^{l+1} K_{0}\left(\xi_{i} z\right) \mathrm{d} z .
$$

The modified Bessel function of the first kind $I_{0}$ has a series representation given by

$$
I_{0}(x)=\sum_{k=0}^{\infty}\left(\frac{x}{2}\right)^{2 k} \frac{1}{(k !)^{2}}
$$

which simplifies $\mathcal{F}_{\sigma_{l}}$ to the final expression

$$
\mathcal{F}_{\sigma_{l}}=-2^{l} \int_{0}^{\infty} z I_{0}(\sqrt{t} z) \prod_{i=1}^{l+1} K_{0}\left(\xi_{i} z\right) \mathrm{d} z
$$

\section{B Inhomogeneous differential equation for the four-loop case}

In this appendix we give an inhomogeneous differential equation

$$
\mathcal{D} \hat{\mathcal{F}}_{\sigma_{4}}=S
$$

satisfied by the four-loop banana Feynman integral $\hat{\mathcal{F}}_{\sigma_{4}}$ (which includes the extra factor (5.2) in the numerator) in the case of generic masses. Operators $\mathcal{D}_{k}$ leading to homogeneous differential equations for $\hat{\mathcal{F}}_{\sigma_{4}}$ have already been given in (5.8). Indeed, here we only present the leading contribution in $z_{i}$ to $\mathcal{D}$, which reads

$$
\begin{aligned}
\mathcal{D}= & -63 \theta_{2}^{2}-416 \theta_{1} \theta_{3}-13 \theta_{2} \theta_{3}+206 \theta_{3}^{2}-180 \theta_{1} \theta_{4}+102 \theta_{2} \theta_{4}+507 \theta_{3} \theta_{4}+180 \theta_{4}^{2} \\
& +596 \theta_{1} \theta_{5}-89 \theta_{2} \theta_{5}-78 \theta_{3} \theta_{5}-429 \theta_{4} \theta_{5}-323 \theta_{5}^{2}+\mathcal{O}\left(z_{i}\right) .
\end{aligned}
$$


The complete expression of this second-order operator $\mathcal{D}$ can be found in a supplementary Mathematica-file on our web page. ${ }^{46}$ Furthermore, the inhomogeneity to $\mathcal{D}$ is given by

$$
\begin{aligned}
S= & \left(-42 z_{1}+168 z_{2}-101 z_{3}+282 z_{4}-139 z_{5}\right) \log \left(z_{1}\right) \log \left(z_{2}\right) \\
& +\left(-416-556 z_{1}+283 z_{2}+105 z_{3}-15 z_{4}-128 z_{5}\right) \log \left(z_{1}\right) \log \left(z_{3}\right) \\
& +\left(-180-180 z_{1}-345 z_{2}-195 z_{3}+540 z_{5}\right) \log \left(z_{1}\right) \log \left(z_{4}\right) \\
& +\left(596+778 z_{1}-106 z_{2}+191 z_{3}-267 z_{4}-273 z_{5}\right) \log \left(z_{1}\right) \log \left(z_{5}\right) \\
& +\left(-13+533 z_{1}+203 z_{2}-21 z_{3}-15 z_{4}-128 z_{5}\right) \log \left(z_{2}\right) \log \left(z_{3}\right) \\
& +\left(102+123 z_{1}+168 z_{2}-195 z_{3}-6 z_{5}\right) \log \left(z_{2}\right) \log \left(z_{4}\right) \\
& +\left(-89-614 z_{1}-539 z_{2}+317 z_{3}-267 z_{4}+273 z_{5}\right) \log \left(z_{2}\right) \log \left(z_{5}\right) \\
& +\left(507+122 z_{1}-477 z_{2}+407 z_{3}-252 z_{4}-139 z_{5}\right) \log \left(z_{3}\right) \log \left(z_{4}\right) \\
& +\left(-78-99 z_{1}-9 z_{2}-491 z_{3}+282 z_{4}+395 z_{5}\right) \log \left(z_{3}\right) \log \left(z_{5}\right) \\
& +\left(-429-65 z_{1}+654 z_{2}-17 z_{3}+252 z_{4}-395 z_{5}\right) \log \left(z_{4}\right) \log \left(z_{5}\right) .
\end{aligned}
$$

\section{Pari/GP script for the equal mass integral}

The Pari/GP script BananaAmplitude.gp ${ }^{47}$ allows to compute the equal mass integral for any given loop order and to any given precision. To explain how one can use the program we want to numerically confirm the special value of the four-loop integral at $s=1$.

After reading the script into GP one has to specify the number of significant digits for internal computations and a variable $N$ that controls to which order the solutions of the differential equations are expanded:

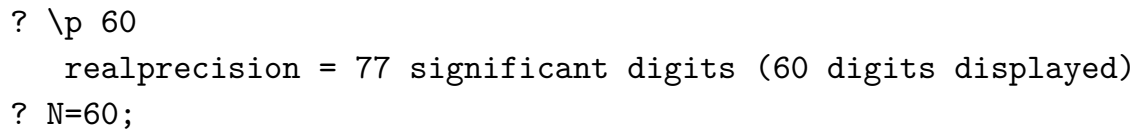

These variables have to be chosen by hand to match the desired accuracy. A linear change in the variable that limits the accuracy results in a linear change of the accuracy in decimal digits. ${ }^{48}$ To generate the $l$-loop integral one has to call the associated function and give the number of loops.

\section{? f=AMPLITUDE (4);}

In this step, the solutions of the differential equation are expanded around all singular points and the integral is analytically continued from $s=0$ to the complete positive real axis. The integral $f(s)$ can now be calculated for any positive $s \neq 1 /(l+1)^{2}$. E.g. to check the special value at $s=1$ we write:

? $f(1)-8 * \mathrm{Pi}^{\sim} 2 * \operatorname{lfun}(\operatorname{lfunmf}(\operatorname{mf} \operatorname{init}([6,4], 0)$, mffrometaquo $([1,2 ; 2,2 ; 3,2 ; 6,2])), 2)$ +4.9173920300900275825221994294591114746 E-53*I

\footnotetext{
${ }^{46}$ http://www.th.physik.uni-bonn.de/Groups/Klemm/data.php

${ }^{47}$ The script can be downloaded on our web page http://www.th.physik.uni-bonn.de/Groups/Klemm/ data.php.

${ }^{48}$ One possibility for checking the accuracy is to evaluate the integral for some $s>1 /(l+1)^{2}$. In this regime the imaginary part has to vanish and this gives a good method to control the accuracy.
} 
Open Access. This article is distributed under the terms of the Creative Commons Attribution License (CC-BY 4.0), which permits any use, distribution and reproduction in any medium, provided the original author(s) and source are credited.

\section{References}

[1] K.G. Chetyrkin and F.V. Tkachov, Integration by parts: the algorithm to calculate $\beta$-functions in 4 loops, Nucl. Phys. B 192 (1981) 159 [INSPIRE].

[2] F.V. Tkachov, A theorem on analytical calculability of four loop renormalization group functions, Phys. Lett. B 100 (1981) 65 [INSPIRE].

[3] A.G. Grozin, Integration by parts: an introduction, Int. J. Mod. Phys. A 26 (2011) 2807 [arXiv: 1104.3993] [INSPIRE].

[4] Y. Zhang, Lecture notes on multi-loop integral reduction and applied algebraic geometry, arXiv: 1612.02249 [INSPIRE].

[5] V.A. Smirnov, Analytic tools for Feynman integrals, Springer, Germany (2012) [INSPIRE].

[6] S. Bauberger, M. Böhm, G. Weiglein, F.A. Berends and M. Buza, Calculation of two-loop self-energies in the electroweak standard model, Nucl. Phys. B Proc. Suppl. 37 (1994) 95 [hep-ph/9406404] [INSPIRE].

[7] R. Bonciani et al., Evaluating a family of two-loop non-planar master integrals for Higgs + jet production with full heavy-quark mass dependence, JHEP 01 (2020) 132 [arXiv: 1907.13156] [INSPIRE].

[8] S. Abreu, M. Becchetti, C. Duhr and R. Marzucca, Three-loop contributions to the $\rho$ parameter and iterated integrals of modular forms, JHEP 02 (2020) 050 [arXiv:1912.02747] [INSPIRE].

[9] S. Laporta, Analytical expressions of 3 and 4-loop sunrise Feynman integrals and 4-dimensional lattice integrals, Int. J. Mod. Phys. A 23 (2008) 5007 [arXiv:0803.1007] [INSPIRE].

[10] L. Adams, C. Bogner, A. Schweitzer and S. Weinzierl, The kite integral to all orders in terms of elliptic polylogarithms, J. Math. Phys. 57 (2016) 122302 [arXiv:1607.01571] [InSPIRE].

[11] J. Broedel, C. Duhr, F. Dulat, R. Marzucca, B. Penante and L. Tancredi, An analytic solution for the equal-mass banana graph, JHEP 09 (2019) 112 [arXiv:1907.03787] [INSPIRE].

[12] M.Y. Kalmykov and B.A. Kniehl, Counting the number of master integrals for sunrise diagrams via the Mellin-Barnes representation, JHEP 07 (2017) 031 [arXiv:1612.06637] [INSPIRE].

[13] T. Bitoun, C. Bogner, R.P. Klausen and E. Panzer, Feynman integral relations from parametric annihilators, Lett. Math. Phys. 109 (2019) 497 [arXiv:1712.09215] [INSPIRE].

[14] L. Adams and S. Weinzierl, Feynman integrals and iterated integrals of modular forms, Commun. Num. Theor. Phys. 12 (2018) 193 [arXiv:1704.08895] [INSPIRE].

[15] J. Ablinger et al., Iterated elliptic and hypergeometric integrals for Feynman diagrams, J. Math. Phys. 59 (2018) 062305 [arXiv:1706.01299] [INSPIRE].

[16] A. Klemm, C. Nega and R. Safari, The l-loop banana amplitude from GKZ systems and relative Calabi-Yau periods, JHEP 04 (2020) 088 [arXiv:1912.06201] [INSPIRE]. 
[17] S. Hosono, A. Klemm, S. Theisen and S.-T. Yau, Mirror symmetry, mirror map and applications to complete intersection Calabi-Yau spaces, Nucl. Phys. B 433 (1995) 501 [hep-th/9406055] [INSPIRE].

[18] A. Klemm, The B-model approach to topological string theory on Calabi-Yau n-folds, in B-model Gromov-Witten theory, E. Clader ed., Trends in Mathematics, Springer, Germany (2018).

[19] C. Bogner and S. Weinzierl, Periods and Feynman integrals, J. Math. Phys. 50 (2009) 042302 [arXiv: 0711.4863] [INSPIRE].

[20] M. Kontsevich and D. Zagier, Periods, in Mathematics unlimited - 2001 and beyond, B. Engquist and W. Schmid, Springer, Germany (2001).

[21] S. Müller-Stach, S. Weinzierl and R. Zayadeh, Picard-Fuchs equations for Feynman integrals, Commun. Math. Phys. 326 (2014) 237 [arXiv:1212.4389] [INSPIRE].

[22] P. Vanhove, The physics and the mixed Hodge structure of Feynman integrals, Proc. Symp. Pure Math. 88 (2014) 161 [arXiv:1401.6438] [INSPIRE].

[23] D.A. Cox, J.B. Little and H.K. Schenck, Toric varieties, Graduate Studies in Mathematics volume 124, American Mathematical Society, Providence U.S.A. (2011).

[24] P. Vanhove, Feynman integrals, toric geometry and mirror symmetry, talk given at the $K M P B$ conference: elliptic integrals, elliptic functions and modular forms in quantum field theory, October 23-26, Zeuthen, Germany (2018) [arXiv:1807.11466] [INSPIRE].

[25] I.M. Gel'fand, A.V. Zelevinsky and M.M. Kapranov, Newton polyhedra of principal A-determinants, Dokl. Akad. Nauk SSSR 308 (1989) 20.

[26] I.M. Gel'fand, A.V. Zelevinsky and M.M. Kapranov, Generalized Euler integrals and A-hypergeometric functions, Adv. Math. 84 (1990) 255.

[27] I.M. Gel'fand, A.V. Zelevinsky and M.M. Kapranov, Hypergeometric functions and toric varieties, Funkt. Anal. Prilozhen. 23 (1989) 12.

[28] E. Nasrollahpoursamami, Periods of Feynman diagrams and GKZ D-modules, arXiv: 1605.04970.

[29] E. Nasrollahpoursamami, Periods of Feynman diagrams, Ph.D. thesis, Caltech, Pasadena, U.S.A. (2017) [INSPIRE].

[30] T.-F. Feng, C.-H. Chang, J.-B. Chen and H.-B. Zhang, GKZ-hypergeometric systems for Feynman integrals, Nucl. Phys. B 953 (2020) 114952 [arXiv:1912.01726] [INSPIRE].

[31] R.P. Klausen, Hypergeometric series representations of Feynman integrals by GKZ hypergeometric systems, JHEP 04 (2020) 121 [arXiv:1910.08651] [INSPIRE].

[32] L. de la Cruz, Feynman integrals as A-hypergeometric functions, JHEP 12 (2019) 123 [arXiv: 1907.00507] [INSPIRE].

[33] K. Schultka, Toric geometry and regularization of Feynman integrals, arXiv:1806.01086 [INSPIRE].

[34] K. Schultka, Microlocal analyticity of Feynman integrals, Ph.D. thesis, Humboldt University, Berlin Germany (2019).

[35] A. Primo and L. Tancredi, On the maximal cut of Feynman integrals and the solution of their differential equations, Nucl. Phys. B 916 (2017) 94 [arXiv:1610.08397] [INSPIRE]. 
[36] A. Primo and L. Tancredi, Maximal cuts and differential equations for Feynman integrals. An application to the three-loop massive banana graph, Nucl. Phys. B 921 (2017) 316 [arXiv: 1704.05465] [INSPIRE].

[37] C. Bogner and S. Weinzierl, Feynman graph polynomials, Int. J. Mod. Phys. A 25 (2010) 2585 [arXiv: 1002.3458] [INSPIRE].

[38] V.V. Batyrev, Dual polyhedra and mirror symmetry for Calabi-Yau hypersurfaces in toric varieties, J. Alg. Geom. 3 (1994) 493.

[39] T. Coates, A. Corti, S. Galkin, V. Golyshev and A. Kasprzyk, Fano varieties and extremal laurent polynomials: A collaborative research blog, http://coates.ma.ic.ac.uk/fanosearch/, December (2012).

[40] V.V. Batyrev and L.A. Borisov, On Calabi-Yau complete intersections in toric varieties, alg-geom/9412017 [INSPIRE].

[41] D. H. Bailey, J. M. Borwein, D. Broadhurst and M. Glasser, Elliptic integral evaluations of bessel moments and applications, J. Phys. A 41 (2008) 205203.

[42] D. Broadhurst, Multiple Zeta values and modular forms in quantum field theory, in Computer algebra in quantum field theory: integration, summation and special functions, C. Schneider and J. Blümlein eds., Springer, Germany (2013) [INSPIRE].

[43] D. Broadhurst and D.P. Roberts, Quadratic relations between Feynman integrals, PoS (LL2018) 053 [INSPIRE].

[44] Y. Zhou, Wronskian factorizations and Broadhurst-Mellit determinant formulae, Commun. Num. Theor. Phys. 12 (2018) 355 [arXiv:1711.01829] [INSPIRE].

[45] Y. Zhou, Some algebraic and arithmetic properties of Feynman diagrams, talk given at the $K M P B$ conference: elliptic integrals, elliptic functions and modular forms in quantum field theory, October 23-26, Zeuthen, Germany (2018) [arXiv: 1801.05555] [INSPIRE].

[46] Y. Zhou, Q-linear dependence of certain Bessel moments, arXiv:1911.04141 [INSPIRE].

[47] D. Broadhurst, Feynman integrals, L-series and Kloosterman moments, Commun. Num. Theor. Phys. 10 (2016) 527 [arXiv: 1604.03057] [INSPIRE].

[48] J. Fresán, C. Sabbah and J.D. Yu, Hodge theory of Kloosterman connections, arXiv: 1810.06454.

[49] J. Fresán, C. Sabbah and J.-D. Yu, Quadratic relations between Bessel moments, arXiv: 2006.02702 [INSPIRE].

[50] H.A. Verrill, Sums of squares of binomial coefficients, with applications to Picard-Fuchs equations, math/0407327.

[51] J.M. Borwein and B. Salvy, A proof of a recurrence for bessel moments, Exp. Math. 17 (2008) 223.

[52] M. Bronstein, T. Mulders and J.-A. Weil, On symmetric powers of differential operators, in the proceedings of the 1997 International Symposium on Symbolic and Algebraic Computation (ISSAC97), Kihei, U.S.A. (1997).

[53] Y. Zhou, Wick rotations, Eichler integrals, and multi-loop Feynman diagrams, Commun. Num. Theor. Phys. 12 (2018) 127 [arXiv:1706.08308] [INSPIRE].

[54] S. Bloch, M. Kerr and P. Vanhove, A Feynman integral via higher normal functions, Compos. Math. 151 (2015) 2329 [arXiv:1406.2664] [INSPIRE]. 
[55] C. Peters, J. Top and M. van der Vlugt, The Hasse zeta function of a K3 surface related to the number of words of weight 5 in the Melas codes, J. Reine Angew. Math. 432 (1992) 151.

[56] K. Hulek and H. Verrill, On modularity of rigid and nonrigid Calabi-Yau varieties associated to the root lattice A4, Nagoya Math. J. 179 (2005) 103.

[57] P. Candelas, X. de la Ossa, M. Elmi and D. Van Straten, A one parameter family of Calabi-Yau manifolds with attractor points of rank two, JHEP 10 (2020) 202 [arXiv: 1912.06146] [INSPIRE].

[58] K. Bönisch, Modularity, periods and quasiperiods at special points in Calabi-Yau moduli spaces, Master thesis, University of Bonn, Bonn, Germany (2020), http://www.th.physik.uni-bonn.de/Groups/Klemm/data.php.

[59] A. Libgober, Chern classes and the periods of mirrors, Math. Res. Lett. 6 (1999) 141.

[60] S. Hosono, Local mirror symmetry and type IIA monodromy of Calabi-Yau manifolds, Adv. Theor. Math. Phys. 4 (2000) 335 [hep-th/0007071] [InSPIRE].

[61] H. Iritani, Ruan's conjecture and integral structures in quantum cohomology, in New developments in algebraic geometry, integrable systems and mirror symmetry, S. Hosono et al. eds., Advanced Studies Pure Mathematics volume 59, Mathematical Society Japan, Tokyo, Japan (2010).

[62] L. Katzarkov, M. Kontsevich and T. Pantev, Hodge theoretic aspects of mirror symmetry, in From Hodge theory to integrability and TQFT tt*-geometry, R.Y. Donagi and K. Wendland, Proceedings of Symposia in Pure Mathematics volume 78, AMS, Providence U.S.A. (2008).

[63] S. Galkin, V. Golyshev and H. Iritani, Gamma classes and quantum cohomology of Fano manifolds: Gamma conjectures, Duke Math. J. 165 (2016) 2005 [arXiv:1404.6407] [INSPIRE].

[64] F. Hirzebruch, Topological methods in algebraic geometry, $3^{\text {rd }}$ edition, Springer, Germany (1966).

[65] P.S. Aspinwall, D-branes on Calabi-Yau manifolds, in the proceedings of the Theoretical Advanced Study Institute in Elementary Particle Physics (TASI 2003), June 2-27, Boulder, U.S.A. (2003) [hep-th/0403166] [INSPIRE].

[66] M. Kontsevich, Homological algebra of mirror symmetry, in the proceedings of the International Congress of Mathematicians 19944 (ICM 1994), August 3-11, Zurich, Switzerland (1994).

[67] P. Seidel and R. Thomas, Braid group actions on derived categories of coherent sheaves, Duke Math. J. 108 (2001) 37.

[68] A. Klemm, B. Lian, S.S. Roan and S.-T. Yau, Calabi-Yau fourfolds for M-theory and F-theory compactifications, Nucl. Phys. B 518 (1998) 515 [hep-th/9701023] [INSPIRE].

[69] N. Cabo Bizet, A. Klemm and D. Vieira Lopes, Landscaping with fluxes and the E8 Yukawa Point in F-theory, arXiv: 1404.7645 [INSPIRE].

[70] K. Oguiso, On algebraic fiber space structures on a Calabi-Yau 3-fold, Int. J. Math. 4 (1993) 439.

[71] H. Iritani, Asymptotics of the banana Feynman amplitudes at the large complex structure limit, arXiv:2011.05901 [INSPIRE]. 
[72] M. Kerr, Unipotent extensions and differential equations (after Bloch-Vlasenko), arXiv:2008.03618.

[73] O. Schnetz, Numbers and functions in quantum field theory, Phys. Rev. D 97 (2018) 085018 [arXiv: 1606.08598] [INSPIRE].

[74] C. Itzykson and J. Zuber, Quantum field theory, International Series In Pure and Applied Physics, McGraw-Hill, New York U.S.A. (1980).

[75] F. Brown and O. Schnetz, Proof of the zig-zag conjecture, arXiv:1208.1890 [INSPIRE].

[76] F. Brown and D. Doryn, Framings for graph hypersurfaces, arXiv:1301.3056 [INSPIRE].

[77] F. Brown and O. Schnetz, A K3 in $\phi^{4}$, Duke Math. J. 161 (2012) 1817 [arXiv: 1006.4064] [INSPIRE].

[78] E. Panzer and O. Schnetz, The Galois coaction on $\phi^{4}$ periods, Commun. Num. Theor. Phys. 11 (2017) 657 [arXiv: 1603.04289] [INSPIRE].

[79] O. Schnetz, The Galois coaction on the electron anomalous magnetic moment, Commun. Num. Theor. Phys. 12 (2018) 335 [arXiv:1711.05118] [INSPIRE].

[80] S. Laporta, High-precision calculation of the 4-loop contribution to the electron $g-2$ in QED, Phys. Lett. B 772 (2017) 232 [arXiv:1704.06996] [inSPIRE].

[81] M.-x. Huang, A. Klemm, J. Reuter and M. Schiereck, Quantum geometry of del Pezzo surfaces in the Nekrasov-Shatashvili limit, JHEP 02 (2015) 031 [arXiv:1401.4723] [INSPIRE].

[82] F. Fischbach, A. Klemm and C. Nega, WKB method and quantum periods beyond genus one, J. Phys. A 52 (2019) 075402 [arXiv: 1803.11222] [InSPIRE].

[83] S. Bloch and P. Vanhove, The elliptic dilogarithm for the sunset graph, J. Number Theor. 148 (2015) 328 [arXiv:1309.5865] [INSPIRE].

[84] A. Klemm, W. Lerche and P. Mayr, K3 fibrations and heterotic type-II string duality, Phys. Lett. B 357 (1995) 313 [hep-th/9506112] [InSPIRE].

[85] M. Mariño and G.W. Moore, Counting higher genus curves in a Calabi-Yau manifold, Nucl. Phys. B 543 (1999) 592 [hep-th/9808131] [InSPIRE].

[86] A. Klemm and M. Mariño, Counting BPS states on the enriques Calabi-Yau, Commun. Math. Phys. 280 (2008) 27 [hep-th/0512227] [INSPIRE].

[87] T.W. Grimm, A. Klemm, M. Mariño and M. Weiss, Direct integration of the topological string, JHEP 08 (2007) 058 [hep-th/0702187] [INSPIRE].

[88] S. Groote, J.G. Korner and A.A. Pivovarov, On the evaluation of a certain class of Feynman diagrams in x-space: Sunrise-type topologies at any loop order, Annals Phys. 322 (2007) 2374 [hep-ph/0506286] [INSPIRE]. 